\title{
Exceptional points in tunable superconducting resonators
}

\author{
Matti Partanen $\odot,{ }^{1,2, *}$ Jan Goetz, ${ }^{1}$ Kuan Yen Tan, ${ }^{1}$ Kassius Kohvakka, ${ }^{1}$ Vasilii Sevriuk, ${ }^{1}$ Russell E. Lake,, 3 \\ Roope Kokkoniemi, ${ }^{1}$ Joni Ikonen, ${ }^{1}$ Dibyendu Hazra, ${ }^{1}$ Akseli Mäkinen, ${ }^{1}$ Eric Hyyppä, ${ }^{1}$ Leif Grönberg, ${ }^{4}$ \\ Visa Vesterinen, ${ }^{1,4}$ Matti Silveri, ${ }^{1,5}$ and Mikko Möttönen ${ }^{1,4, \dagger}$ \\ ${ }^{1}$ QCD Labs, QTF Centre of Excellence, Department of Applied Physics, Aalto University, P.O. Box 13500, FI-00076 Aalto, Finland \\ ${ }^{2}$ Walther-Meißner-Institut, Bayerische Akademie der Wissenschaften, DE-85748 Garching, Germany \\ ${ }^{3}$ National Institute of Standards and Technology, Boulder, Colorado 80305, USA \\ ${ }^{4}$ VTT Technical Research Centre of Finland Ltd, P.O. Box 1000, FI-02044 VTT, Finland \\ ${ }^{5}$ Research Unit of Nano and Molecular Systems, University of Oulu, P.O. Box 3000, FI-90014 Oulu, Finland
}

(Received 17 May 2019; revised manuscript received 9 August 2019; published 7 October 2019)

\begin{abstract}
Superconducting quantum circuits are potential candidates to realize a large-scale quantum computer. The envisioned large density of integrated components, however, requires a proper thermal management and control of dissipation. To this end, it is advantageous to utilize tunable dissipation channels and to exploit the optimized heat flow at exceptional points (EPs). Here, we experimentally realize an EP in a superconducting microwave circuit consisting of two resonators. The EP is a singularity point of the effective Hamiltonian, and corresponds to critical damping with the most efficient heat transfer between the resonators without back and forth oscillation of energy. We observe a crossover from underdamped to overdamped coupling across the EP by utilizing photonassisted tunneling as an in situ tunable dissipative element in one of the resonators. These methods can be used to obtain fast dissipation, for example, for initializing qubits to their ground states. In addition, these results pave the way for thorough investigation of parity-time symmetry and the spontaneous symmetry breaking at the EP in superconducting quantum circuits operating at the level of single energy quanta.
\end{abstract}

DOI: 10.1103/PhysRevB.100.134505

\section{INTRODUCTION}

Systems with effective non-Hermitian Hamiltonians have been actively studied in various setups in recent years [1-7]. They show many intriguing properties such as singularities in their energy spectra [8-13]. A square-root singularity point in the parameter space of a non-Hermitian matrix is referred to as an exceptional point (EP) if the eigenvalues coalesce $[12,13]$. Previously, EPs have been shown to emerge, for example, in nonsuperconducting microwave circuits, laser physics, quantum phase transitions, and atomic and molecular physics [12,13]. The fascinating effects of EPs include the disappearance of the beating Rabi oscillations [14], chiral states in microwave systems [15], and spontaneous symmetry breaking in systems with parity- and time-reversal $(\mathcal{P} \mathcal{T})$ symmetry [16-19]. In the quantum regime, $\mathcal{P} \mathcal{T}$-symmetric systems may show features that are different from the semiclassical predictions, such as new phases owing to quantum fluctuations $[19,20]$. Despite the active research on EPs, they have not been sufficiently investigated in superconducting microwave circuits to date [21].

\footnotetext{
*matti.t.partanen@aalto.fi

${ }_{\dagger}^{\dagger}$ mikko.mottonen@aalto.fi
}

Published by the American Physical Society under the terms of the Creative Commons Attribution 4.0 International license. Further distribution of this work must maintain attribution to the author(s) and the published article's title, journal citation, and DOI.
Superconducting microwave circuits provide an ideal platform to realize various quantum technological devices, such as ultrasensitive photon detectors and counters [22-25], and potentially even a large-scale quantum computer [26,27], or a quantum simulator [28] in the framework of circuit quantum electrodynamics [29,30]. Notably, superconducting qubits have been shown to approach the required coherence times [31,32] for quantum error correction [33,34]. However, despite the tremendous interest in superconducting microwave circuits in recent years [35-37], there are still many issues to be solved before a fully functional quantum computer is realizable. For example, the precise engineering of energy flows between different parts of the circuit in scalable architectures is of utmost importance since heat is a typical source of decoherence in qubits [38,39]. In many error correction codes, qubits are repeatedly initialized, which requires fast and efficient cooling schemes [40-42]. It is also of great importance to reset the qubit readout resonators, for example, using pulse shaping $[43,44]$. One very promising method for absorbing energy and, hence, initializing qubits to their ground state is based on resonators with tunable dissipation [45].

The recently developed quantum-circuit refrigerator (QCR) [46,47] provides great potential for both qubit initialization and thermal management since it enables tunability of energy dissipation rates over several orders of magnitude in a superconducting microwave resonator [48]. The operation of the QCR relies on inelastic tunneling of electrons through a normal-metal-insulator-superconductor (NIS) junction [49]. The tunneling electrons can absorb 
(a)

$$
\mathrm{R} 1
$$
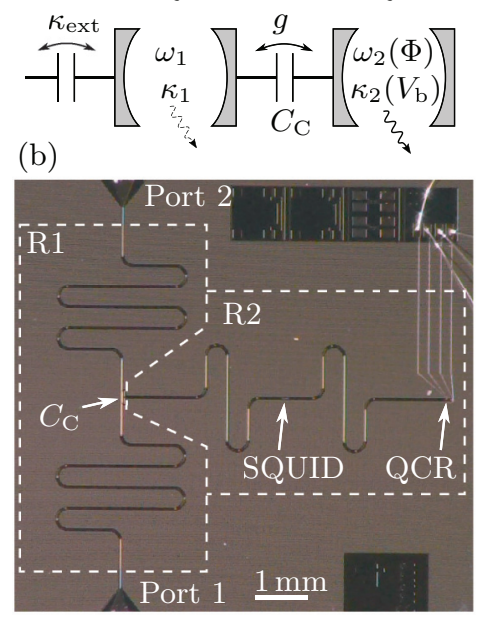

FIG. 1. Sample structure. (a) The sample consists of two capacitively coupled resonators, R1 and R2, which are presented as analogous cavities with coupling strength $g$. The primary resonator $\mathrm{R} 1$ has a fixed dissipation rate $\kappa_{1}$ and angular frequency $\omega_{1}$ whereas the dissipative resonator $\mathrm{R} 2$ has a tunable rate $\kappa_{2}\left(V_{\mathrm{b}}\right)$ controlled by a QCR, and angular frequency $\omega_{2}(\Phi)$ tuned with a SQUID. The coupling strength to external ports is denoted by $\kappa_{\text {ext }}$. (b) Optical micrograph of the sample. The transmission coefficient $S_{21}$ is measured from port 1 to port 2. (c) False-color scanning electron micrograph of the QCR together with a schematic control circuit. The QCR consists of normal-metal $(\mathrm{N})$ and superconducting (S) electrodes separated by an insulator (I). The QCR is operated with bias voltage $V_{\mathrm{b}}$, and the electron temperature of the normal metal is obtained from voltage $V_{\text {th }}$ and current $I_{\text {th }}$. (d) The operation principle of a SINIS junction. The occupied states in the superconductor density of states are shown in blue, the occupation of the normal metal is given by the Fermi distribution shown in orange, and the empty states are shown in gray with energy $E$ on the vertical axis. The Fermi levels of the superconducting electrodes (dashed lines) are shifted by applying a voltage $V_{\mathrm{b}}$. The black arrows indicate elastic tunneling, and blue arrows inelastic tunneling with photon absorption. The red dashed arrows show photon emission that is suppressed due to lack of available unoccupied states on the other side of the tunneling barrier.

or emit photons to a resonator which allows to control the coupling strength to a low-temperature bath in situ. This tunable coupling strength arising from a broadband environment has also been shown to induce a Lamb shift [48].

In this work, we utilize tunable dissipation to realize EPs, which correspond to critical coupling between two superconducting microwave resonators. To this end, we investigate a circuit consisting of two coupled resonators, one of which is equipped with NIS junctions and a flux-tunable resonance frequency (Fig. 1). We denote the NIS junctions and the normalmetal island that is capacitively coupled to the resonator as a QCR. Thanks to the voltage-tunable dissipation within the QCR and the flux-tunable resonance frequency, an EP arises in the Hamiltonian that describes the modes of the coupled resonator system. We investigate the emergence of the EP using frequency and dissipation as control parameters (Fig. 2) and verify its properties experimentally by measuring the microwave transmission coefficient (Fig. 3). The maximum
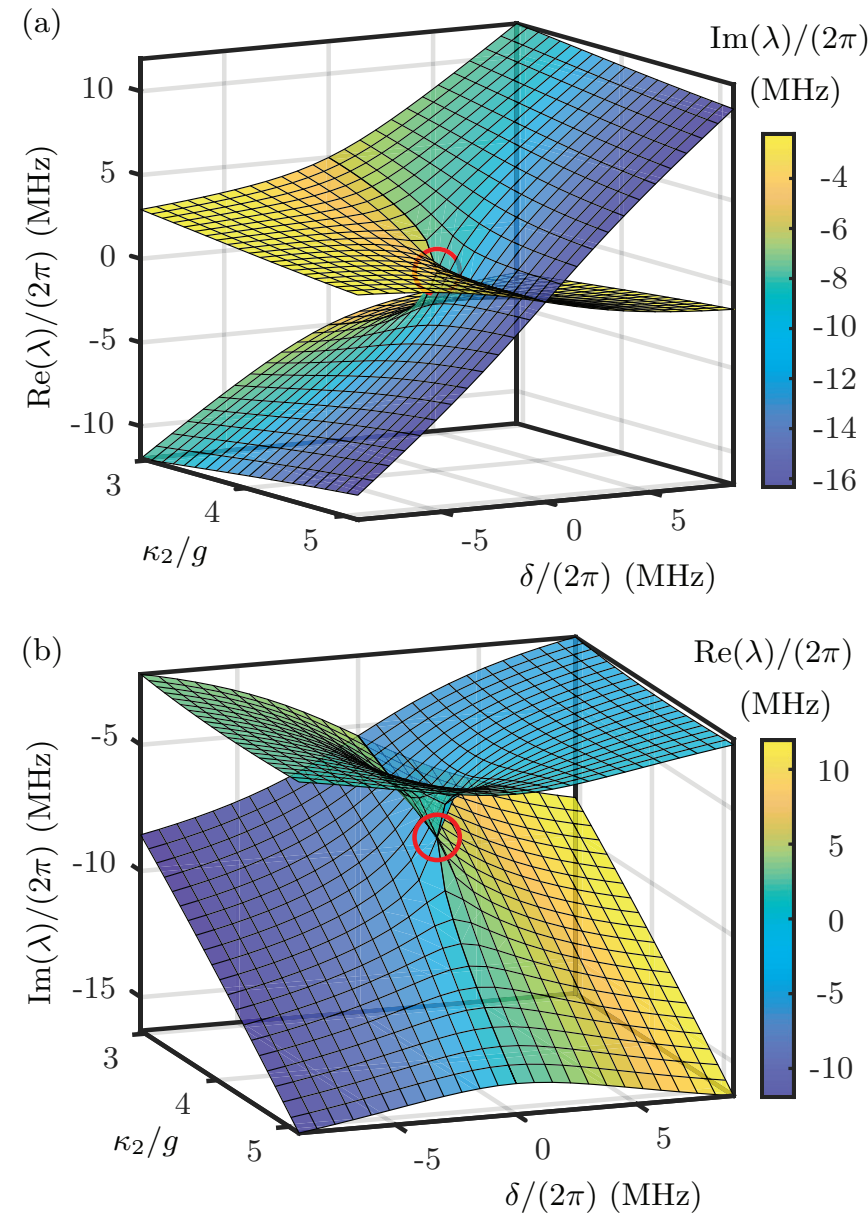

FIG. 2. Eigenvalues of the effective Hamiltonian and the exceptional point. (a) Calculated mode frequency shift $\operatorname{Re}(\lambda)$ with respect to the uncoupled mode frequency of $\mathrm{R} 1$, and (b) negative value of the mode decay rate $-\operatorname{Im}(\lambda)$ as functions of the decay rate $\kappa_{2}$ and frequency detuning $\delta$. The figure shows both $\lambda_{+}$and $\lambda_{-}$calculated according to Eq. (2) with the experimental coupling strength $g /(2 \pi)=7.2 \mathrm{MHz}$ and decay rate $\kappa_{1} /(2 \pi)=260 \mathrm{kHz}$. The EP is located approximately at $\kappa_{2}=4 g$, and $\delta=0$ as indicated with the red circle. The panels (a) and (b) can be compared with each other with the help of the colors which denote $\operatorname{Im}(\lambda)$ in (a) and $\operatorname{Re}(\lambda)$ in (b).

dissipation rate given by the coupling strength can be reached at the EP (Fig. 4). Furthermore, we demonstrate temporal control of the damping rates by utilizing voltage pulses at the QCR (Fig. 5). By adding voltage-tunable dissipation to a system of two resonators with tunable coupling, our approach provides a conceptually higher level of control compared to the commonly studied systems which only have tunable coupling between the resonators [42,50-57]. Hence, our work demonstrates a platform to control the dissipation and, consequently, local heat transport between neighboring nodes in a quantum electrical circuit. In addition to thermal management within superconducting multiqubit systems, these methods may be applicable to thermally assisted quantum annealing [58] and to studies of the eigenstate thermalization hypothesis in many-body quantum problems [59]. Furthermore, our work is an important step toward thorough investigation of $\mathcal{P} \mathcal{T}$-symmetric systems at the quantum level 


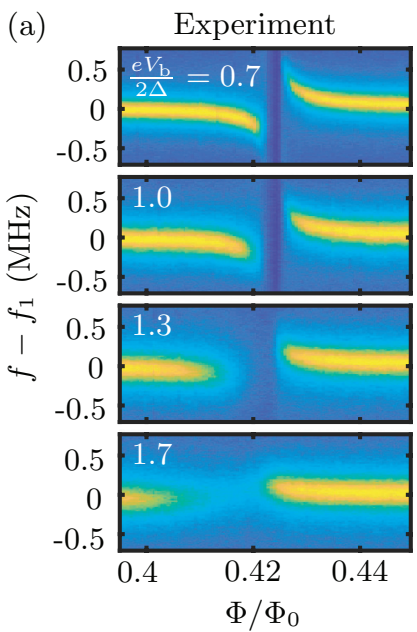

$\left|S_{21}\right|$

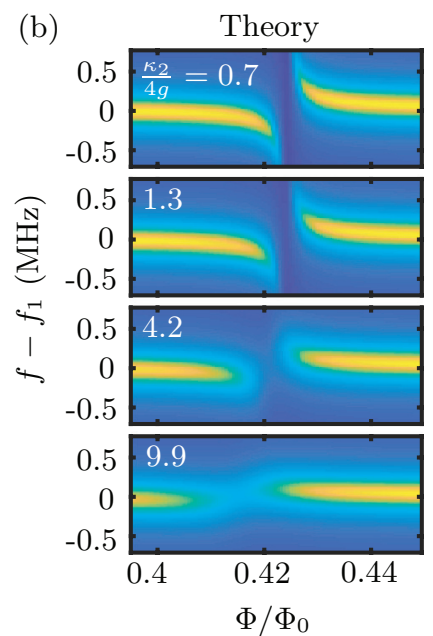

FIG. 3. Scattering parameter of sample A. (a) Experimental and (b) theoretical transmission amplitudes as functions of frequency and magnetic flux through the SQUID. The panels in (a) show the crossing of the second mode of R1 and the first mode of R2 at different bias voltages, and the panels in (b) show the corresponding theoretical results at different coupling strengths, as indicated in the figure. The $\mathrm{EP}$ is obtained at $e V_{\mathrm{b}} /(2 \Delta) \approx 1$, where the ratio $\kappa_{2} /(4 g)$ crosses unity. The maximum in each panel is normalized to unity, and the frequency axes are shifted by the resonance frequency $f_{1}=$ $5.223 \mathrm{GHz}$ in good agreement with the simulated frequency $f_{1}=$ $5.2 \mathrm{GHz}$. The input power at port 1 is approximately $-100 \mathrm{dBm}$.

that can be realized with circuit quantum electrodynamics architectures [21,60].

This paper is structured as follows: We introduce the experimental samples in Sec. II, describe the EPs in Sec. III, and present the experimental results in Sec. IV. The results are discussed in Sec. V. Extended measurement results are shown in Appendix A, electron tunneling through NIS junctions is described in Appendices B and C, quantum-mechanical and classical models for our samples are discussed in detail in Appendices D-I, and experimental techniques are presented in Appendices $\mathrm{J}-\mathrm{L}$.

\section{EXPERIMENTAL SAMPLES}

Our samples consist of two coplanar waveguide resonators, R1 and R2, which are capacitively coupled to each other, as depicted in Figs. 1(a) and 1(b). The resonator R1 has a fixed fundamental frequency at $2.6 \mathrm{GHz}$. This mode does not couple strongly to the resonator R2 owing to the voltage node in the middle of the resonator $\mathrm{R} 1$ where the coupling capacitor $C_{\mathrm{C}}$ is located. Therefore, we focus on the second mode of $\mathrm{R} 1$, with frequency $f_{1}=\omega_{1} /(2 \pi)=5.2 \mathrm{GHz}$, which has a voltage antinode at the coupling point of the resonators. The resulting capacitive coupling between the resonators has a strength $g /(2 \pi)=7.2 \mathrm{MHz}$. In contrast to $\mathrm{R} 1$, the resonator R2 has a flux-tunable resonance frequency $\omega_{2}(\Phi)$ owing to a superconducting quantum interference device (SQUID), and a voltage-tunable loss rate $\kappa_{2}\left(V_{\mathrm{b}}\right)$ owing to the QCR. Here, $\Phi$ and $V_{\mathrm{b}}$ are the magnetic flux threading the SQUID loop and the voltage bias of the QCR, respectively. The theoretical
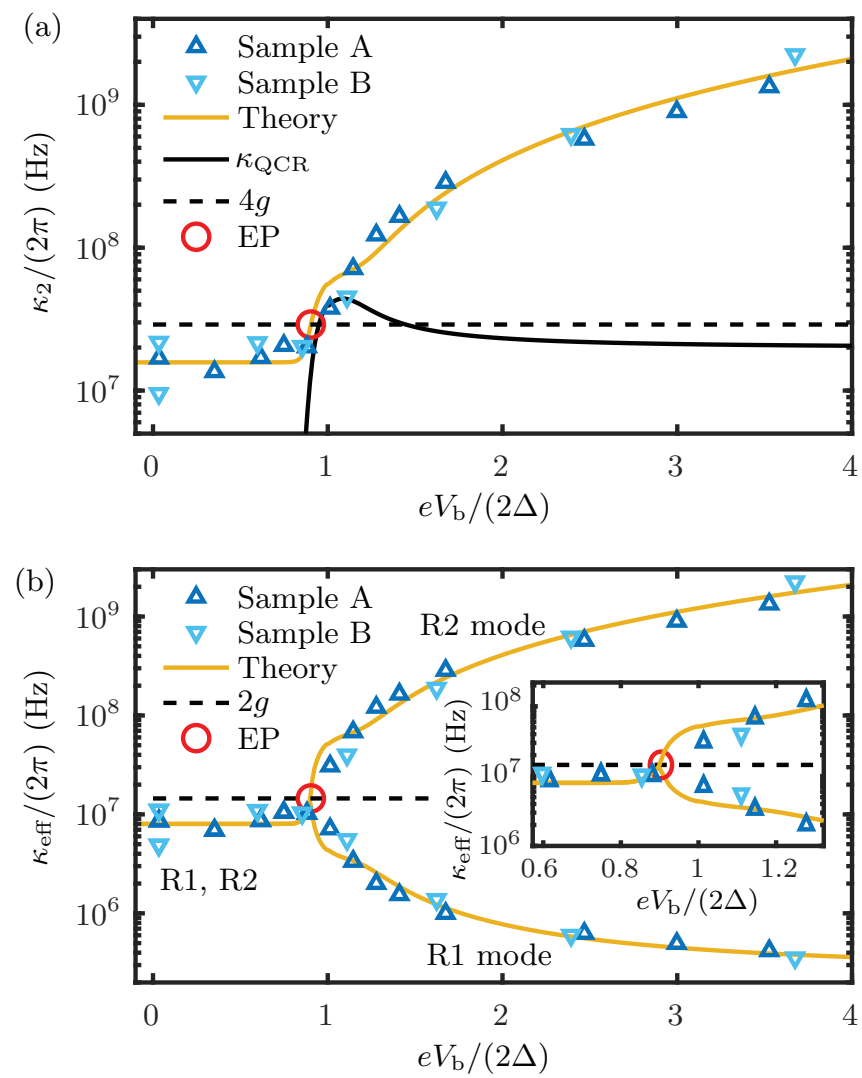

FIG. 4. Transition rates. (a) Extracted decay rates of the bare resonator $\mathrm{R} 2, \kappa_{2}$, for samples $\mathrm{A}$ and $\mathrm{B}$ as functions of the bias voltage together with the theoretical model (Appendix H). The EP is obtained at the intersection of $\kappa_{2}$ and the critical coupling $4 g$. Furthermore, the figure shows the theoretical coupling strength of the $\mathrm{QCR}, \kappa_{\mathrm{QCR}}$, without taking dephasing and other voltage-dependent losses into account. Here, the flux is approximately $\Phi / \Phi_{0}=0.4$, and the frequency $5.2 \mathrm{GHz}$. The probe power at the input of the device is $-100 \mathrm{dBm}$ for sample $\mathrm{A}$ and $-115 \mathrm{dBm}$ for sample $\mathrm{B}$. The uncertainty of the data points is of the same order as the marker size. (b) Experimental and theoretical effective decay rates of the coupled system $\kappa_{\text {eff }}=-2 \operatorname{Im}\left(\lambda_{ \pm}\right)$obtained from $\kappa_{2}$ at zero detuning using Eq. (2). The two branches at high voltages correspond to $\lambda_{+}$and $\lambda_{-}$with the modes located predominantly in one of the resonators as indicated. The maximum decay rate for $\mathrm{R} 1$ is obtained at the EP. The damping rates of the modes are equal at $e V_{\mathrm{b}} /(2 \Delta)<1$ due to hybridization. The inset shows the effective damping rate in the voltage regime near the exceptional point.

background for the QCR is discussed in Appendix B and Ref. [49]. The inductance of the SQUID and, hence, also the resonance frequency of $\mathrm{R} 2$ are periodic in the flux with a period of a flux quantum $\Phi_{0}=h /(2 e)$. Consequently, due to the coupling of the resonators, R1 also shows flux-dependent features. We show the QCR in Fig. 1(c) and schematically present its operation principle in Fig. 1(d). The difference in photon absorption and emission rates originates from the gap of $2 \Delta$ in the density of states of the superconductor, and the difference can be utilized to cool down quantum circuits $[46,49]$. We study two samples with different $\mathrm{R} 2$ resonator lengths: sample A $(12 \mathrm{~mm})$ and sample B $(13 \mathrm{~mm})$. The $\mathrm{R} 1$ resonator has a length of $24 \mathrm{~mm}$ in both samples. See 


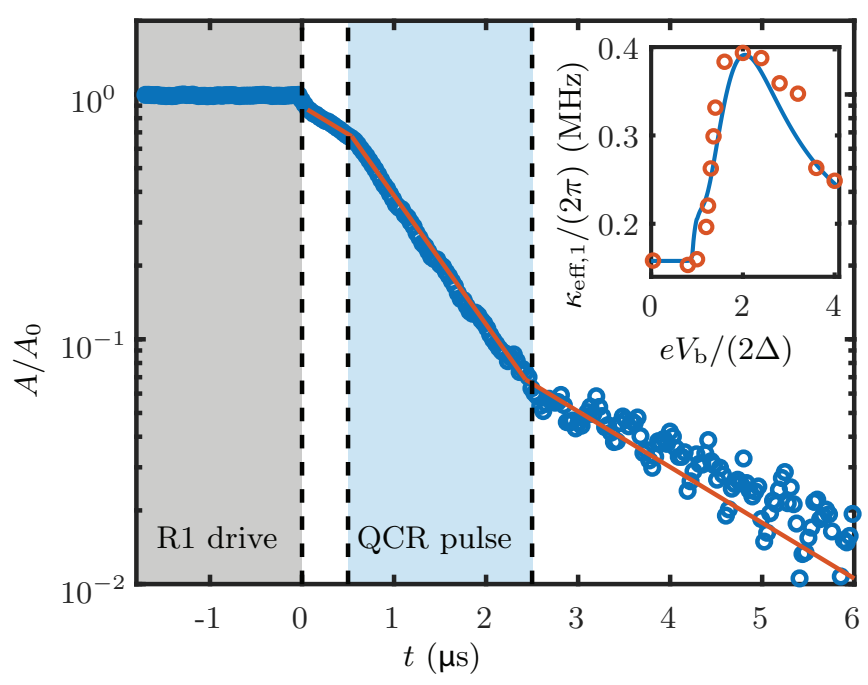

FIG. 5. Energy dissipation of sample B as a function of time. Measured voltage amplitude $A(t)$ of the resonator R1 normalized with $A(0)=A_{0}$ (markers). We apply microwave drive to $\mathrm{R} 1$ at the resonance frequency for $40 \mu \mathrm{s}$ and switch it off at time $t=0$. At $t \approx 0.5 \mu \mathrm{s}$, we send nominally rectangular 2 - $\mu$ s-long voltage pulse with the amplitude $e V_{\mathrm{b}} /(2 \Delta) \approx 2.4$ to the QCR, which results in increased energy dissipation. After the pulse, the dissipation decreases approximately to its original value, as shown by the model (solid line). The magnetic flux through the SQUID is shifted from the mode crossing by approximately $0.015 \times \Phi_{0}$ yielding a detuning of $200 \mathrm{MHz}$ (see Appendix I). The inset shows the experimental (markers) and theoretical (solid line) decay rate of the R1 mode during the pulse as a function of the pulse amplitude. The input power is approximately $-105 \mathrm{dBm}$.

Appendices E, J, and $\mathrm{K}$ for sample parameters, fabrication, and measurement setup, respectively.

\section{EXCEPTIONAL POINTS}

To study exceptional points, we utilize two control parameters in the effective Hamiltonian of the system: we use the voltage-tunable dissipation rate $\kappa_{2}\left(V_{\mathrm{b}}\right)$ and the flux-tunable detuning between the resonators $\delta(\Phi)=\omega_{2}(\Phi)-\omega_{1}$. We study the system consisting of the two resonators in a frame rotating with a frequency corresponding to the uncoupled mode frequency of R1. Thus, the excitations of the system can be described with the effective non-Hermitian Hamiltonian in matrix form operating on a vector $\psi=(A, B)^{\mathrm{T}}$ where $A$ and $B$ are field amplitudes in R1 and R2, respectively,

$$
H=\left(\begin{array}{cc}
-i \frac{\kappa_{1}}{2} & g \\
g & \delta-i \frac{\kappa_{2}}{2}
\end{array}\right),
$$

where $\kappa_{1}$ is the decay rate of the resonator R1. This effective Hamiltonian is analogous with the actual quantummechanical Hamiltonian operating on the corresponding Hilbert space, and it can be derived from the Lindblad master equation (see Appendix D and Refs. [56,61]). The eigenvalues of $H$ can be written as

$$
\lambda_{ \pm}=\frac{1}{4}\left(2 \delta-i \kappa_{1}-i \kappa_{2} \pm s\right)
$$

and the corresponding eigenvectors are

$$
\psi_{ \pm}=\left(\frac{-2 \delta-i \kappa_{1}+i \kappa_{2} \pm s}{4 g}, 1\right)^{\mathrm{T}}
$$

where $s=\sqrt{\left(2 \delta+i \kappa_{1}-i \kappa_{2}\right)^{2}+16 g^{2}}$. Thus, the eigenvalues and eigenvectors coalesce when the square-root term $s$ vanishes resulting in an EP. Consequently, there is only a single eigenvalue and, importantly, there is also only a single eigenvector. The EP occurs at $\left|\kappa_{2}-\kappa_{1}\right|=4 g$, and $\delta=0$. In our samples $\kappa_{1} \ll \kappa_{2}$, which we verify by measuring the internal quality factor of the primary resonator R1 with R2 far detuned at $\Phi=\Phi_{0} / 2$. From the quality factor, we extract a loss rate $\kappa_{1} \lesssim 2 \pi \times 260 \mathrm{kHz}$ for both samples which is substantially lower than the extracted value of $\kappa_{2}$, as discussed below. Consequently, the condition for the EP simplifies to $\kappa_{2}=4 g$.

To visualize the system singularity, i.e., the EP, we show the real and imaginary parts of $\lambda_{ \pm}$in Fig. 2. The eigenvalues form a self-intersecting Riemann surface in the parameter space of $\kappa_{2}$ and $\delta$. The imaginary part corresponds to mode decay, and real part to mode frequency deviation from the uncoupled mode frequency of $\mathrm{R} 1$. Our system consisting of the resonators $\mathrm{R} 1$ and $\mathrm{R} 2$ can be considered as a single damped harmonic oscillator, where the energy oscillates between the two resonators, as discussed in Appendix D. In the underdamped case $\kappa_{2}<4 g$, the modes have an equal decay rate at zero detuning, and there is an anticrossing of the mode frequencies. In contrast, in the overdamped case $\kappa_{2}>4 g$, there is an anticrossing in the mode decay rates as a function of the detuning, and the mode frequencies are equal at zero detuning. Consequently, one of the modes remains lossy whereas the other one has a low decay rate at different detunings.

Let us connect the meaning of this critical point to the efficiency of energy transfer between the two resonators. In terms of coupled dissipative systems, the EP separates the system between the overdamped and underdamped regimes being the point of critical coupling. It follows from the dynamics of the coupled system that at this point, the energy is transferred between the two resonators optimally fast without back and forth oscillation [42,56]. In particular, the rate of heat transfer at zero detuning is given by $\kappa_{\text {eff }}=-2 \operatorname{Im}\left(\lambda_{ \pm}\right) \approx$ $\kappa_{2}\left\{1 \mp \operatorname{Re}\left[\sqrt{1-\left(4 g / \kappa_{2}\right)^{2}}\right]\right\} / 2$. Here, the branch with the upper signs corresponds to a mode located predominantly in the primary resonator $\mathrm{R} 1$, and the branch with the lower ones in the dissipative resonator $\mathrm{R} 2$. Consequently, by reaching the EP at $\kappa_{2}=4 g$, we operate our sample at a point of optimally efficient heat transfer out of R1. The effective coupling strength in the limit $\kappa_{1} \rightarrow 0$ is also relevant for Purcell filters [62].

\section{EXPERIMENTAL OBSERVATIONS}

To explore the dissipative dynamics of the two coupled resonators, we measure the flux- and frequency-dependent scattering parameter $S_{21}$ describing the transmission from port 1 to port 2 for different bias voltages using a vector network analyzer. We tune the magnetic flux in a range where the frequency of R2 crosses that of R1. As shown in Fig. 3(a), we observe a transition from an anticrossing 
into a single mode already indicating the presence of an EP between these regimes. A broader range of bias voltages is shown in Appendix A. To generate a quantitative description of our system, which is required for the investigation of EPs, we simulate the scattering coefficient using an analytical circuit model, as shown in Fig. 3(b). Here, we model the SQUID as a flux-tunable inductor, and the QCR as an effective resistance $R_{\text {eff }}$ describing the dissipation (see Appendix I). The theoretical model in Fig. 3 is in very good agreement with the experimental results. Consequently, we can extract the experimental damping rates of the dissipative resonator $\mathrm{R} 2$ as a function of the bias voltage using the circuit model. In addition to the damping rates, we extract the coupling capacitance $C_{\mathrm{C}}$ and the critical current of the SQUID, $I_{\mathrm{c}}$, from the theoretical model (see Appendices F, G, and H).

To demonstrate the presence of an EP, we show the extracted damping rates of the bare resonator $\mathrm{R} 2$ in the absence of R1 in Fig. 4(a) as functions of the bias voltage for samples $\mathrm{A}$ and $\mathrm{B}$. The damping rate $\kappa_{2}$ is approximately equal in both samples and its value can be tuned by approximately two orders of magnitude. At low bias voltages $e V_{\mathrm{b}} /(2 \Delta)<1$, the rate $\kappa_{2}$ is below $4 g$, and at $e V_{\mathrm{b}} /(2 \Delta)>1$ the damping rate exceeds $4 g$. The continuity of the theoretical curve guarantees a crossing at the critical coupling and, thus, the existence of the EP. We describe the origin of the tunable damping rates using a theoretical model that contains the photon absorption and emission at the QCR given by the rate $\kappa_{\mathrm{QCR}}$, as well as constant internal losses $\kappa_{\text {int,2, }}$, and voltage-dependent residual losses $\kappa_{\mathrm{r}, 2}$. We have designed our sample in such a way that $\kappa_{\mathrm{QCR}}$ covers the critical damping rate, and thus the losses originating from the QCR are sufficient to realize the EP. The underlying analysis is based on the excellent agreement between theory and experiment found for similar devices in earlier experiments [48]. We can accurately obtain the damping rate $\kappa_{\mathrm{QCR}}$ shown in Fig. 4 using the measured electron temperatures of the normal-metal island (Appendix C). At higher voltages, $\kappa_{2}$ is dominated by the residual damping coefficient, as discussed in Appendix G. This damping coefficient includes dephasing, quasiparticle losses, and resistive losses, and we extract its value based on the experimental rates.

In Fig. 4(b), we show the damping rates of the coupled circuit defined using Eq. (2) as $\kappa_{\text {eff }}=-2 \operatorname{Im}\left(\lambda_{ \pm}\right)$. The maximum energy decay rate for the mode in R1 is obtained at the EP as discussed above, and it is given by $\kappa_{\max , 1}=$ $\max \left[-2 \operatorname{Im}\left(\lambda_{+}\right)\right] \approx \kappa_{2} / 2=2 g$. Thus, in the optimal case, the decay rate is limited by the coupling strength between the resonators, a scenario related to the quantum speed limit. We do not observe a distinctively large increase in the damping rate of R1 when approaching the EP with increasing bias voltages since $\kappa_{2}$ is already relatively close to the optimal value $4 g$ at low voltages. Furthermore, $\kappa_{2}$ is very sensitive to the voltage near the EP.

In Fig. 5, we study the time dependence of the damping rates to demonstrate the temporal control we have over the system. To this end, we operate the system with a finite detuning $\delta$. For our samples, the finite detuning provides a convenient measurement point with relatively low damping rates, thus allowing us to clearly observe the changes in their values even when the system is operated at the single-photon level. Furthermore, at this operation point the measured damping rates are less affected by the experimental inaccuracy of the magnetic flux compared to zero detuning. To extract the time- and voltage-dependent damping rates, we measure the ring-down of R1 in time domain. During the ring-down, we apply a nominally rectangular $2-\mu$ s-long voltage pulse to the QCR to study the effect of the induced dissipation from $\mathrm{R} 2$. The finite detuning reduces the sensitivity of the damping rates to the nonidealities in the pulse reaching the sample. The pulse increases the dissipation rate, as expected. After the pulse, the dissipation returns approximately to an equal level with that before the pulse. The instantaneous response of the damping rates to the applied voltages shows that we can control the dissipation on a timescale substantially shorter than $1 \mu \mathrm{s}$. The QCR can be operated even at timescales in the range of $10 \mathrm{~ns}$ [63]. Furthermore, the dependence of the damping rate on the pulse amplitude follows the theory, as shown in the inset. The position of the peak is shifted to higher voltages as compared to Figs. 4(a) and 4(b) owing to the detuning. The good agreement of the time traces and the fact that the system returns back to its intrinsic relaxation rate quickly after turning off the voltage of the QCR shows that we can reliably control the dissipative dynamics within the parameter space spanned by $\kappa_{2}$ and $\delta$.

\section{SUMMARY AND DISCUSSION}

We have experimentally realized an exceptional point (EP) in a superconducting microwave circuit consisting of two coupled resonators. We study the presence of the EP by observing a transition from an avoided crossing to single modulating resonance frequency. This point corresponds to the maximum heat transfer between the two resonators without back and forth oscillation of the energy. The measurement results are in very good agreement with our theoretical model. The circuit is based on a QCR, which operates as a voltage-tunable dissipator, thus enabling the investigation of the crossover from an underdamped to critically damped and further to overdamped circuit. In addition to the realization of an EP, the circuit also behaves as a frequency- and voltage-tunable heat sink for quantum electric circuits that can be applied, for example, in quantum information processing for initializing qubits to their ground state by absorbing energy [45]. The tunability of the damping rate enables one to obtain the fastest possible photon absorption allowed by a given coupling coefficient.

In the future, it is interesting to further investigate the EP by modifying the circuit design. By introducing tunnel junctions to both resonators, one obtains a continuous line of EPs instead of an isolated singularity point. Incorporating qubits also enables the investigation and utilization of the EP with single energy quanta. Furthermore, one can investigate resonators with an additional coupling circuit realized with a SQUID or a qubit [52,54] in addition to the tunable coupling realized with frequency detuning and a tunable damping realized with a QCR that are employed here. Moreover, the use of several microwave resonators will result in a more versatile parameter space [64], and hence yields an interesting platform for studying fundamental physics. Dynamic encircling of the EP with topological energy transfer [3,9] can be realized with superconducting resonators in a straightforward manner using standard microwave techniques. It requires fast tuning 
of the magnetic field, which can be realized by fabricating a flux bias line on the chip. Topological energy transfer with microwave pulses may provide an asset for applications in quantum information processing and other quantum technological devices. In addition, EPs are suitable for investigating $\mathcal{P} \mathcal{T}$ symmetry on the level of single microwave photons. Here, superconducting circuits provide an attractive architecture owing to the ability to design system parameters yielding, for example, ultrastrong- and deep-strong-coupling regimes $[21,65]$. Furthermore, we envision EPs as candidates to realize nonreciprocal signal routing beneficial for active quantum circuits $[60,66,67]$.

Note added. Recently, we became aware of Ref. [68] investigating EPs in superconducting circuits with a different operation principle compared to our system. Our work is fully independent of this reference.

\section{ACKNOWLEDGMENTS}

We thank A. A. Clerk for discussions, and J. Govenius and M. Jenei for assistance. We acknowledge the provision of facilities and technical support by Aalto University at OtaNano-Micronova Nanofabrication Centre. We acknowledge the funding from the European Research Council under Consolidator Grant No. 681311 (QUESS), and Marie Skłodowska-Curie Grant No. 795159, the Academy of Finland through its Centres of Excellence Program (Projects No. 312300 and No. 312059) and Grants No. 265675, No. 305237, No. 305306, No. 308161, No. 312300, No. 314302, and No. 316551), the European Union via the Quantum Flagship project QMiCS (Grant No. 820505), the Vilho, Yrjö and Kalle Väisälä Foundation, the Technology Industries of Finland Centennial Foundation, the Jane and Aatos Erkko Foundation, the Alfred Kordelin Foundation, and the Emil Aaltonen Foundation.

\section{APPENDIX A: MEASUREMENTS IN A BROAD RANGE OF VOLTAGES AND FLUXES}

We show experimentally and theoretically obtained scattering parameter $\left|S_{21}\right|$ over a broad range of bias voltages applied at the QCR and magnetic fluxes through the SQUID in Figs. 6 and 7. The measurements are in good agreement with the theoretical model.

\section{APPENDIX B: QUANTUM-CIRCUIT REFRIGERATOR}

We use a QCR to absorb and emit photons in the resonator $\mathrm{R} 2$. The resonator transition rate from the occupation number $m$ to $m^{\prime}$ can be written as [49]

$$
\Gamma_{m, m^{\prime}}(V)=M_{m m^{\prime}}^{2} \frac{2 R_{\mathrm{K}}}{R_{\mathrm{T}}} \sum_{\tau= \pm 1} \vec{F}\left[\tau e V+\hbar \omega_{2}\left(m-m^{\prime}\right)\right]
$$

where $V=V_{\mathrm{b}} / 2, R_{\mathrm{T}}$ is the single-junction tunneling resistance, $M_{m m^{\prime}}$ is the corresponding matrix element, $R_{\mathrm{K}}=$ $h / e^{2} \approx 25.8 \mathrm{k} \Omega$ is the von Klitzing constant, and the normalized rate for forward tunneling is given by

$$
\vec{F}(E)=\frac{1}{h} \int_{-\infty}^{\infty} d E^{\prime} n_{\mathrm{S}}\left(E^{\prime}\right)\left[1-f\left(E^{\prime}, T_{\mathrm{S}}\right)\right] f\left(E^{\prime}-E, T_{\mathrm{N}}\right),
$$
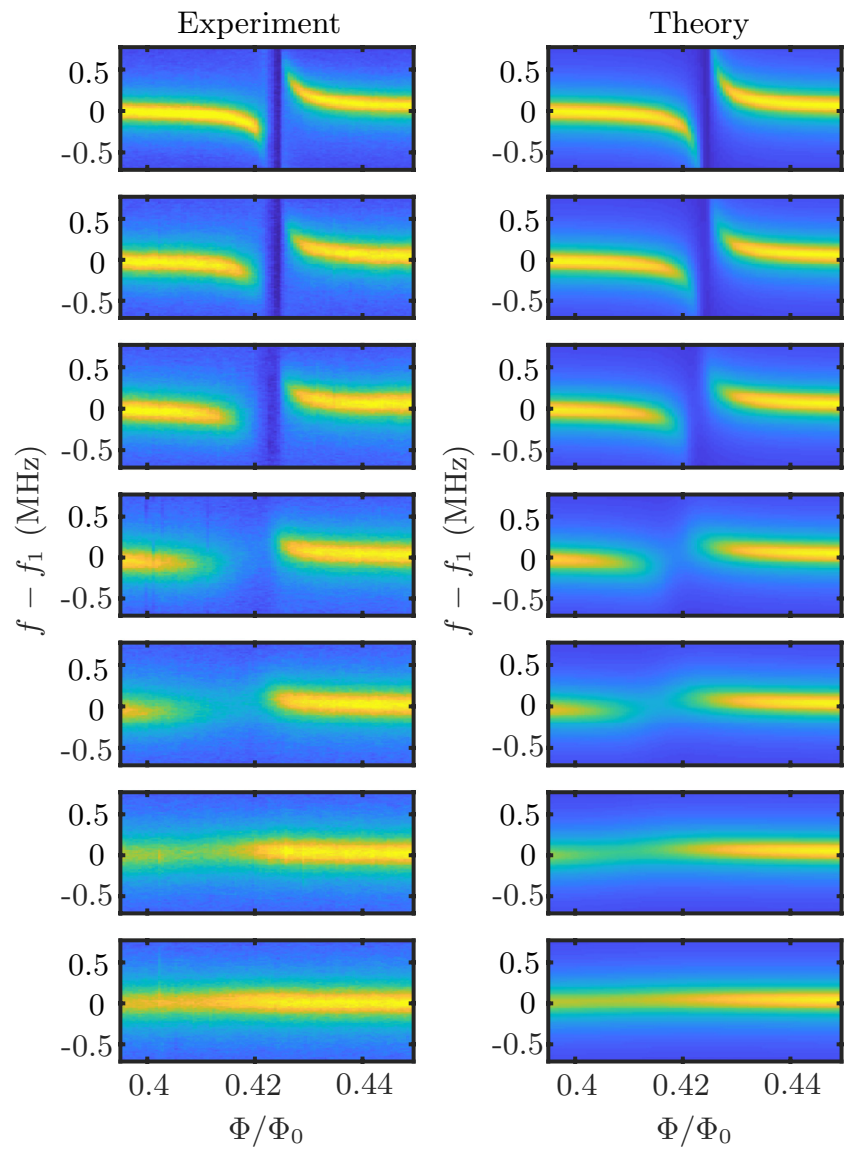

$\left|S_{21}\right|$

FIG. 6. Scattering parameter of sample A. Measured and simulated scattering parameter $\left|S_{21}\right|$ as a function of frequency and flux for different bias voltages. The bias voltages from top to bottom are $e V_{\mathrm{b}} /(2 \Delta)=0.0,1.0,1.1,1.4,1.7,2.5$, and 3.5. Maximum value in each panel is normalized separately to unity. The input power at port 1 is approximately $-100 \mathrm{dBm}$. See Fig. 11 for the fitted parameters and Fig. 4 for the corresponding transition rates.

where $f(E, T)=1 /\left\{\exp \left[E /\left(k_{\mathrm{B}} T\right)\right]+1\right\}$ is the Fermi-Dirac distribution, $k_{\mathrm{B}}$ is the Boltzmann constant, and the density of states in a superconductor can be expressed with the help of the Dynes parameter $\gamma_{D}$ as

$$
n_{\mathrm{S}}(E)=\left|\operatorname{Re}\left(\frac{E / \Delta+i \gamma_{\mathrm{D}}}{\sqrt{\left(E / \Delta+i \gamma_{\mathrm{D}}\right)^{2}-1}}\right)\right| .
$$

The matrix element describing the transition can be written in terms of the generalized Laguerre polynomials $L_{n}^{l}(\rho)$ as [49]

$$
M_{m, m^{\prime}}^{2}= \begin{cases}e^{-\rho} \rho^{m-m^{\prime}} \frac{m^{\prime} !}{m !}\left[L_{m^{\prime}}^{m-m^{\prime}}(\rho)\right]^{2}, & m \geqslant m^{\prime} \\ e^{-\rho} \rho^{m^{\prime}-m} \frac{m !}{m^{\prime} !}\left[L_{m^{\prime}}^{m^{\prime}-m}(\rho)\right]^{2}, & m<m^{\prime}\end{cases}
$$

where $\rho=\pi \alpha_{\mathrm{c}}^{2} /\left(\omega_{2} C_{1} x_{2} R_{\mathrm{K}}\right)$ is an environmental parameter, $C_{1}$ is the capacitance per unit length of the coplanar waveguide, $2 x_{2}$ is the length of the resonator $\mathrm{R} 2$, and the capacitance fraction $\alpha_{\mathrm{c}}$ is given in terms of the capacitance between the normal-metal island and the center conductor $C_{\mathrm{N}}$, and the single-junction capacitance $C_{\mathrm{j}}$ as $\alpha_{\mathrm{c}}=C_{\mathrm{N}} /\left(C_{\mathrm{N}}+4 C_{\mathrm{j}}\right)$. In the equations above, we have neglected the effects owing to 

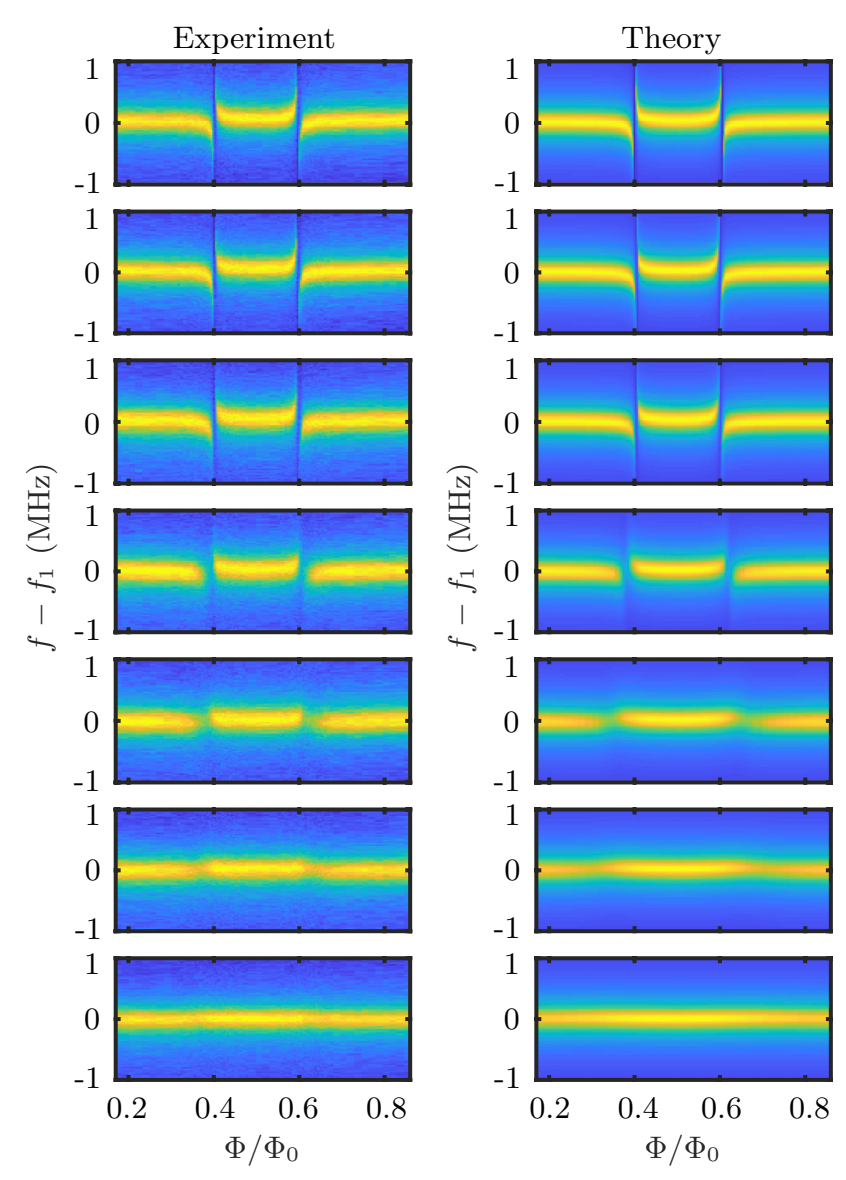

$\left|S_{21}\right|$

FIG. 7. Scattering parameter of sample B. Measured and simulated scattering parameter $\left|S_{21}\right|$ as a function of frequency and flux for different bias voltages. The bias voltages from top to bottom are $e V_{\mathrm{b}} /(2 \Delta)=0.0,0.6,1.1,1.6,2.4,3.7$, and 6.2. The maximum value in each panel is normalized to unity. The input power at port 1 is approximately $-115 \mathrm{dBm}$. At $e V_{\mathrm{b}} /(2 \Delta)=6.2$, the effective resistance obtains the value of $30 \Omega$ and the critical current $I_{\mathrm{c}} / I_{\mathrm{c}, 0} \approx$ 0.3. See Fig. 11 for the the other fitted parameters and Fig. 4 for the corresponding transition rates.

the charging of the normal-metal island since the capacitance of the island is relatively large. Furthermore, the rates for single-photon transitions can be expressed as [49]

$$
\begin{aligned}
& \Gamma_{m, m-1}=\kappa_{\mathrm{QCR}}\left(N_{\mathrm{QCR}}+1\right) m, \\
& \Gamma_{m, m+1}=\kappa_{\mathrm{QCR}} N_{\mathrm{QCR}}(m+1),
\end{aligned}
$$

where $\kappa_{\mathrm{QCR}}$ denotes the coupling strength of the QCR, and the Bose-Einstein distribution at the effective temperature of the electron tunneling $T_{\mathrm{QCR}}$ is given by

$$
N_{\mathrm{QCR}}=\frac{1}{\exp \left(\frac{\hbar \omega_{2}}{k_{\mathrm{B}} T_{\mathrm{QCR}}}\right)-1},
$$

where

$$
T_{\mathrm{QCR}}=\frac{\hbar \omega_{2}}{k_{\mathrm{B}}}\left\{\ln \left[\frac{\sum_{\tau= \pm 1} \vec{F}\left(\tau e V+\hbar \omega_{2}\right)}{\sum_{\tau= \pm 1} \vec{F}\left(\tau e V-\hbar \omega_{2}\right)}\right]\right\}^{-1} .
$$

These equations are derived by defining

$$
\kappa_{\mathrm{QCR}}=\frac{\Gamma_{m, m-1}}{m}-\frac{\Gamma_{m, m+1}}{m+1} .
$$

\section{APPENDIX C: ELASTIC TUNNELING IN NORMAL-METAL-INSULATOR-SUPERCONDUCTOR JUNCTIONS}

Typically, the elastic tunneling is the dominating tunneling process in NIS junctions. It can be utilized for temperature control of the normal-metal electrons [69-71], and for precise thermometry down to millikelvin temperatures [71,72]. Recently, NIS junctions have also been utilized in a realization of a quantum heat valve [73], and phase-coherent caloritronics [74]. Figure 8 shows the measured current-voltage characteristics and normal-metal temperatures.

The electric current through a single NIS junction can be written as [71,75]

$$
\begin{aligned}
I(V)= & \frac{1}{e R_{\mathrm{T}}} \int_{0}^{\infty} n_{\mathrm{S}}(E)\left[f\left(E-e V, T_{\mathrm{N}}\right)\right. \\
& \left.-f\left(E+e V, T_{\mathrm{N}}\right)\right] d E,
\end{aligned}
$$

where $T_{\mathrm{N}}$ denotes the normal-metal temperature, and $V$ is the voltage across the junction. For a symmetric SINIS structure, we apply a voltage $V_{\mathrm{b}}=2 \mathrm{~V}$. Importantly, this equation has a monotonic dependence on the temperature of the normal metal but only a very weak dependence on the temperature of the superconductor. Thus, we may use NIS junctions as thermometers measuring the electron temperature of the normal metal.

The tunneling electrons transfer heat through the insulating barrier. The average power is given by [71]

$$
P=\frac{1}{e^{2} R_{\mathrm{T}}} \int_{-\infty}^{\infty} n_{\mathrm{S}}(E)(E-e V)\left[f\left(E-e V, T_{\mathrm{N}}\right)-f\left(E, T_{\mathrm{S}}\right)\right] d E .
$$

Based on this equation, we can reduce and increase the temperature of the normal metal. The applied voltage at the SINIS junction produces a total Joule heating power $P=V_{\mathrm{b}} I$, which is unequally divided between the $\mathrm{N}$ and $\mathrm{S}$ electrodes.

\section{APPENDIX D: QUANTUM-MECHANICAL MODEL}

Here, we analyze the temporal evolution of the coupled resonators following Ref. [56]. Alternatively, the dynamics of the system can be solved using, for example, a reconstruction method [61]. The Hamiltonian can be written in the rotatingwave approximation as

$$
\hat{H}_{\mathrm{RWA}}=\hbar \omega_{1} \hat{a}^{\dagger} \hat{a}+\hbar \omega_{2} \hat{b}^{\dagger} \hat{b}+\hbar g\left(\hat{a} \hat{b}^{\dagger}+\hat{a}^{\dagger} \hat{b}\right) .
$$

The first term describes the energy of the primary resonator $\mathrm{R} 1$ with annihilation operator $\hat{a}$, the second term the energy of $\mathrm{R} 2$ with annihilation operator $\hat{b}$, and the third term describes the coupling between the resonators. Here, we have neglected driving. Furthermore, this equation is valid only for a linear resonator. The effects owing to nonlinearity are discussed below. To solve the dynamics of the system, we utilize the Lindblad master equation for the density matrix of the coupled 

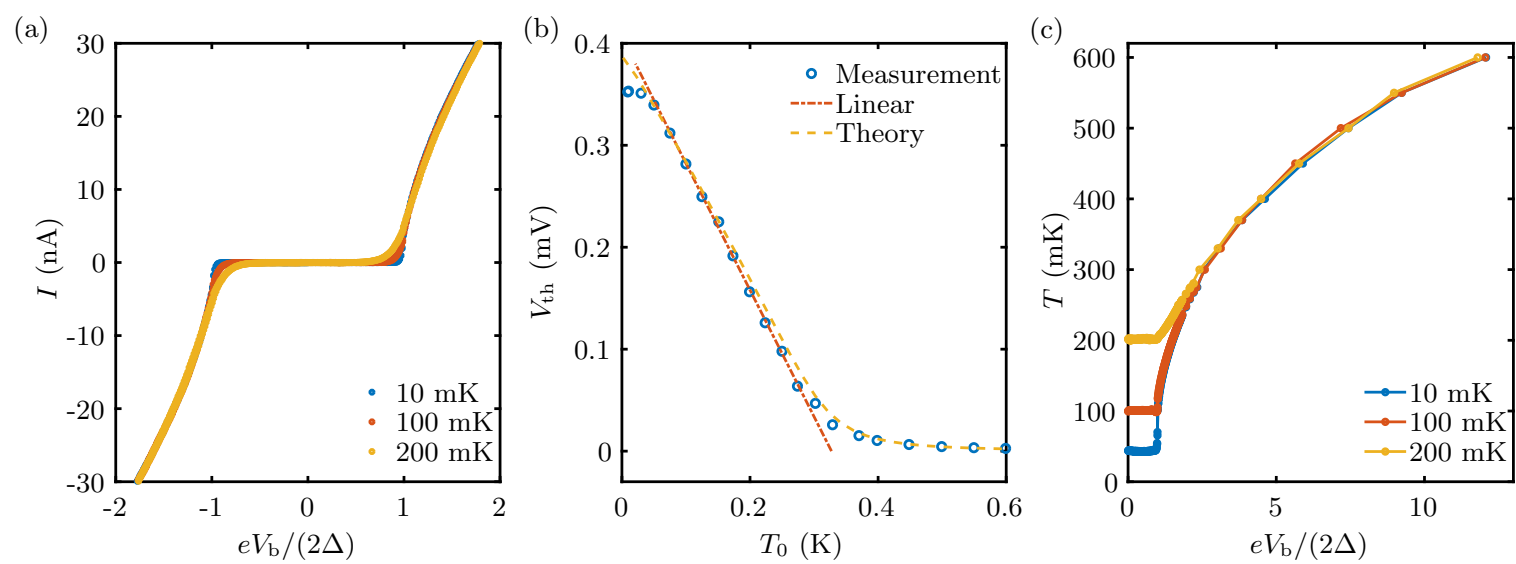

FIG. 8. Current-voltage characteristics and temperature for sample B. (a) Electric current through a SINIS junction as a function of bias voltage at different bath temperatures. (b) Measured thermometer voltage $V_{\text {th }}$ as a function of bath temperature $T_{0}$ at fixed bias current $I_{\text {th }}=$ $17 \mathrm{pA}$, and $V_{\mathrm{b}}=0$. The theoretical curve is calculated using Eq. (C1). We extract the electron temperatures of the normal-metal island using a linear voltage-to-temperature conversion below $300 \mathrm{mK}$, and above that we extract the temperatures from the voltages corresponding to the different experimental bath temperature points. At high temperatures, the low sensitivity reduces the reliability of the extracted island temperatures. (c) Electron temperature of the normal-metal island as a function of bias voltage at different bath temperatures.

system $\hat{\rho}$ as

$$
\frac{d \hat{\rho}}{d t}=-\frac{i}{\hbar}\left[\hat{H}_{\mathrm{RWA}}, \hat{\rho}\right]+\kappa_{1} \mathcal{L}[\hat{a}] \hat{\rho}+\kappa_{2} \mathcal{L}[\hat{b}] \hat{\rho},
$$

where the Lindblad superoperator is defined by $\mathcal{L}[\hat{x}] \hat{\rho}=$ $\hat{x} \hat{\rho} \hat{x}^{\dagger}-\frac{1}{2}\left\{\hat{x}^{\dagger} \hat{x}, \hat{\rho}\right\}$. We can write the resulting equations of motion as [56]

$$
\begin{aligned}
& \frac{d\langle\hat{a}\rangle}{d t}=-i \omega_{1}\langle\hat{a}\rangle-i g\langle\hat{b}\rangle-\frac{\kappa_{1}}{2}\langle\hat{a}\rangle, \\
& \frac{d\langle\hat{b}\rangle}{d t}=-i \omega_{2}\langle\hat{b}\rangle-i g\langle\hat{a}\rangle-\frac{\kappa_{2}}{2}\langle\hat{b}\rangle .
\end{aligned}
$$

We define the resonator fields as $\langle\hat{a}\rangle=A \exp \left(-i \omega_{1} t\right),\langle\hat{b}\rangle=$ $B \exp \left(-i \omega_{1} t\right)$. Consequently, the equations assume the form

$$
\begin{gathered}
\frac{d A}{d t}=-i g B-\frac{\kappa_{1}}{2} A, \\
\frac{d B}{d t}=-i \delta B-i g A-\frac{\kappa_{2}}{2} B,
\end{gathered}
$$

where $\delta=\omega_{2}-\omega_{1}$. These equations can be written in a matrix form as a time-dependent Schrödinger equation

$$
\frac{d}{d t} \psi=-i H \psi
$$

where $\psi=(A, B)^{\mathrm{T}}$, and

$$
H=\left(\begin{array}{cc}
-i \frac{\kappa_{1}}{2} & g \\
g & -i \frac{\kappa_{2}}{2}+\delta
\end{array}\right)
$$

as given in Eq. (1). Here, $H$ is an effective non-Hermitian Hamiltonian scaled by $\hbar$. We note that $H$ operates here on classical field amplitudes instead of quantum states. Nevertheless, Eq. (D7) is analogous to the quantum mechanical Schrödinger equation. The eigenvalues and eigenvectors of $H$ are given by Eqs. (2) and (3) in the main text, respectively, and Fig. 9 shows them as a function of $\kappa_{2}$ for zero detuning.
Equations (D5) and (D6) can also be written as a secondorder differential equation

$$
\frac{d^{2} A}{d t^{2}}+\left(\frac{\kappa_{1}+\kappa_{2}}{2}+i \delta\right) \frac{d A}{d t}+\left(g^{2}+i \delta \frac{\kappa_{1}}{2}+\frac{\kappa_{1} \kappa_{2}}{4}\right) A=0
$$

When the resonators are tuned into resonance, $\delta=0$, we can express Eq. (D9) as

$$
\frac{d^{2} A}{d t^{2}}+\frac{\kappa_{1}+\kappa_{2}}{2} \frac{d A}{d t}+\left(g^{2}+\frac{\kappa_{1} \kappa_{2}}{4}\right) A=0
$$

This equation describes a damped harmonic oscillator corresponding to energy transfer between the resonators R1 and R2 at an angular frequency $\sqrt{g^{2}+\kappa_{1} \kappa_{2} / 4}$. Due to the asymmetric damping rates in the two resonators, the total dissipation rate of the system is time dependent and reaches its maximum value when the excitations are in $\mathrm{R} 2$. The damping ratio is given by

$$
\xi=\frac{\kappa_{1}+\kappa_{2}}{2 \sqrt{4 g^{2}+\kappa_{1} \kappa_{2}}} .
$$

Here, $\kappa_{2}$ is a function of voltage $V_{\mathrm{b}}$, which allows us to examine the transition from an underdamped system $\xi<1$ through critical damping $\xi=1$ to an overdamped system $\xi>1$. Critical damping is obtained when $\left|\kappa_{2}-\kappa_{1}\right|=4 g$. The total damping rate of $\mathrm{R} 1$ is given by $\kappa_{1}=\kappa_{\mathrm{int}, 1}+\kappa_{\mathrm{ext}}$, and of R2 by $\kappa_{2}\left(V_{\mathrm{b}}\right)=\kappa_{\text {int }, 2}+\kappa_{\mathrm{QCR}}\left(V_{\mathrm{b}}\right)+\kappa_{\mathrm{r}, 2}\left(V_{\mathrm{b}}\right)$, where $\kappa_{\text {int }, 1 / 2}$ denote the internal losses, $\kappa_{\text {ext }}$ the losses to the external measurement circuit, $\kappa_{\mathrm{QCR}}\left(V_{\mathrm{b}}\right)$ the photon-assisted tunneling in Eq. (B8), and $\kappa_{\mathrm{r}, 2}\left(V_{\mathrm{b}}\right)$ the residual voltage-dependent losses in R2. In our samples, $\kappa_{2}\left(V_{\mathrm{b}}\right) \gg \kappa_{1}$ and $g \gg \kappa_{\text {int, } 1} \gg \kappa_{\text {ext }}$, as discussed below. Therefore, we obtain an approximate condition for the critical damping as

$$
\kappa_{2}=4 g \text {. }
$$

The critical damping, which corresponds to the EP, is obtained at $e V_{\mathrm{b}} /(2 \Delta) \approx 1$ where the photon number remains low and, 

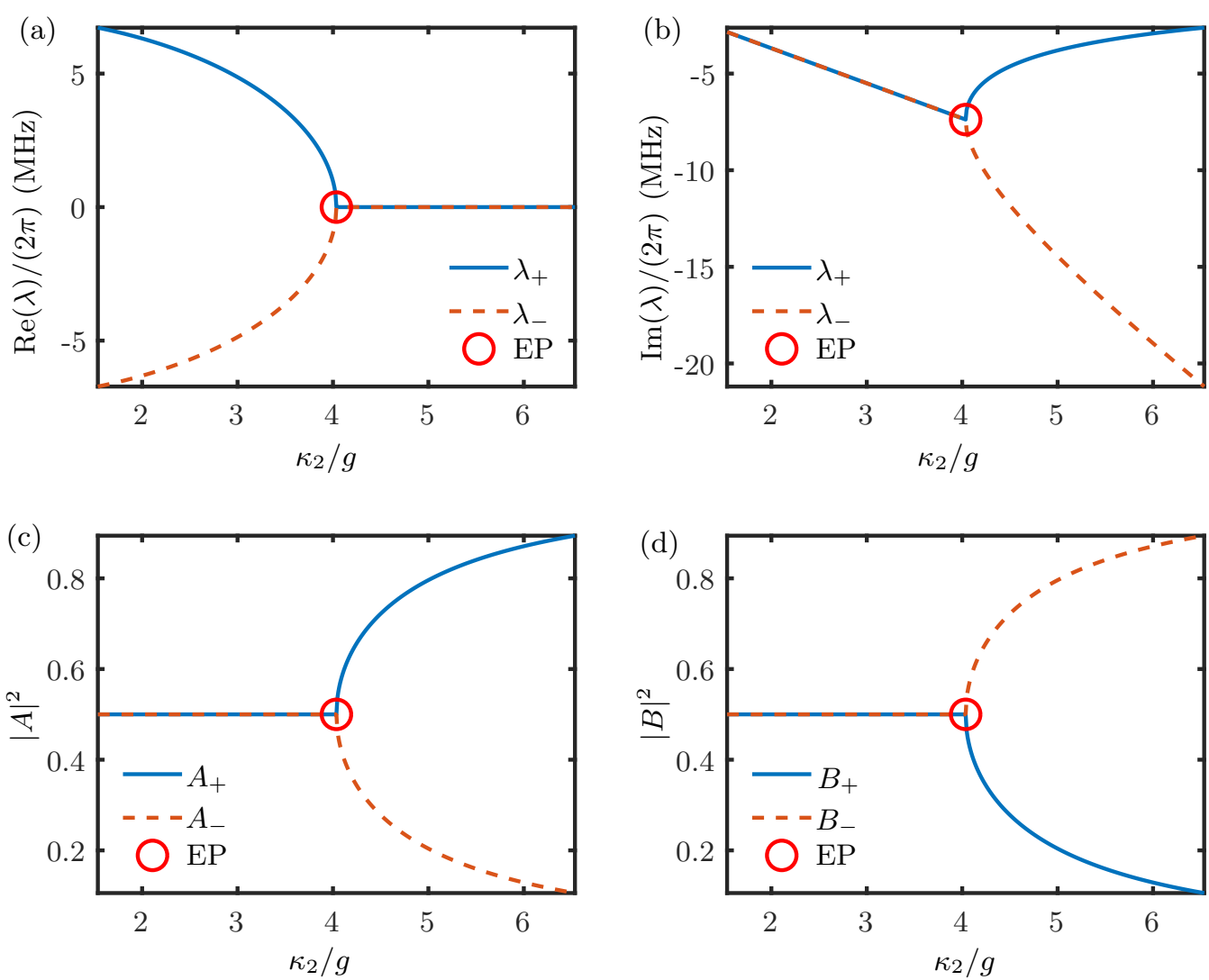

FIG. 9. Eigenvalues and eigenvectors of the effective Hamiltonian. (a) The real part of the eigenvalues corresponding to the frequency shifts from the bare resonator R1 mode frequency as a function of the decay rate $\kappa_{2}$ at zero detuning. (b) The imaginary part of the eigenvalues, the absolute values of which correspond to the decay rates. (c) The squared absolute value of the eigenvector component corresponding to the resonator R1. Here, the amplitude of the eigenvector $\Psi_{ \pm}=\left(A_{ \pm}, B_{ \pm}\right)^{\mathrm{T}}$ is normalized to unity. (d) As (c) but for the eigenvector component corresponding to R2.

therefore, the slight nonlinearity caused by the SQUID is negligible. However, at $e V_{\mathrm{b}} /(2 \Delta)>1$, the QCR generates thermal photons that result in photon-number-dependent losses, as discussed below.

\section{APPENDIX E: SAMPLE PARAMETERS}

The main parameters for the samples are summarized in Table I. The coupling strength between the resonators can be estimated as [76] $g=C_{\mathrm{C}} V_{1} V_{2} / \hbar \approx 2 \pi \times 7.2 \mathrm{MHz}$, where the voltages are given by $V_{i}=\sqrt{\hbar \omega_{1} /\left(2 x_{i} C_{1}\right)}, i=1,2$, the angular frequency of the second mode of the resonator R1 is $\omega_{1} /(2 \pi) \approx 5.223 \mathrm{GHz}$ for samples A and $\mathrm{B}$, and $C_{1}$ is the capacitance per unit length. Consequently, the critical damping is obtained with $\kappa_{2}=4 g \approx 2 \pi \times 29 \mathrm{MHz}$. The external quality factor corresponding to the leakage from the resonator R1 to the transmission line through the capacitances $C_{\mathrm{TL}}$ is given by [77] $Q_{\mathrm{ext}}=2 x_{1} C_{1} /\left(4 Z_{\mathrm{L}} \omega_{1} C_{\mathrm{TL}}^{2}\right) \approx 9 \times 10^{5}$. Consequently, the corresponding damping rate is $\kappa_{\mathrm{ext}}=\omega_{1} / Q_{\mathrm{ext}} \approx 2 \pi \times$ $6 \mathrm{kHz}$. The loaded quality factor of the second mode of R1 is approximately $Q_{\mathrm{L}}=2 \times 10^{4}$, when the resonators are far detuned (Fig. 10). Thus, the internal losses in R1 dominate over the losses to the transmission line $Q_{\mathrm{int}} \approx Q_{\mathrm{L}}$. Furthermore, we obtain the damping rate $\kappa_{1}=\omega_{1} / Q_{\mathrm{L}} \approx 2 \pi \times 300 \mathrm{kHz}$. The real part of the complex wave propagation coefficient, $\gamma=$ $\alpha+i \beta$, describes the damping in the waveguide, and it can be calculated as [77] $\alpha=n_{\mathrm{m}} \pi /\left(4 x_{1} Q_{\mathrm{int}}\right) \approx 7 \times 10^{-3} \mathrm{~m}^{-1}$, where $n_{\mathrm{m}}$ is the mode number such that $n_{\mathrm{m}}=2$ denotes the second mode. The internal losses without the photon-assisted tunneling in the QCR are somewhat higher in the resonator R2 than in R1 since the design and fabrication of the QCR and the SQUID have not been optimized for low loss rates. The internal loss rate for $\mathrm{R} 2$ can be extracted at zero detuning and zero-bias voltage from the saturation level of extracted $\kappa_{2}$ values since $\kappa_{\mathrm{int}, 1} \ll \kappa_{2}(0)$, and hence the losses in R2 dominate over those in R1. We obtain from the circuit model the internal loss rate for $\mathrm{R} 2$ as $\kappa_{\mathrm{int}, 2} \approx \kappa_{2}(0)=2 \pi \times 16 \mathrm{MHz}$. We measure a slight temperature and power dependence of the quality factor of R1 as expected in the case of two-level fluctuators dominating the losses [78] (Fig. 10).

The photon number inside R1 when R2 is far detuned can be estimated as [79] $n=4 \Omega_{\mathrm{d}}^{2} / \kappa_{1}^{2} \approx 10$, where the driving strength is given by $\Omega_{\mathrm{d}}=C_{\mathrm{TL}} V_{\mathrm{in}} V_{1} / \hbar$, the input voltage is obtained from the input power as $V_{\text {in }}=\sqrt{P_{\text {in }} Z_{\mathrm{L}}}$, and the input power is $P_{\text {in }} \approx-115 \mathrm{dBm}$. The input power is $-100 \mathrm{dBm}$ for sample A in Figs. 3 and 6, and $-115 \mathrm{dBm}$ for sample B in Fig. 7. We also measure the resonators at different power levels. When $\mathrm{R} 1$ and $\mathrm{R} 2$ are in resonance at $V_{\mathrm{b}}=0$, the total photon number is approximately equally divided between the resonators if $\kappa_{1} \approx \kappa_{2}$. However, in our samples $\kappa_{1}<\kappa_{2}$, especially at $V_{\mathrm{b}}>2 \Delta / e$, and therefore the number of coherent photons is lower in $\mathrm{R} 2$ than in $\mathrm{R} 1$. When the $Q$ factor of the 

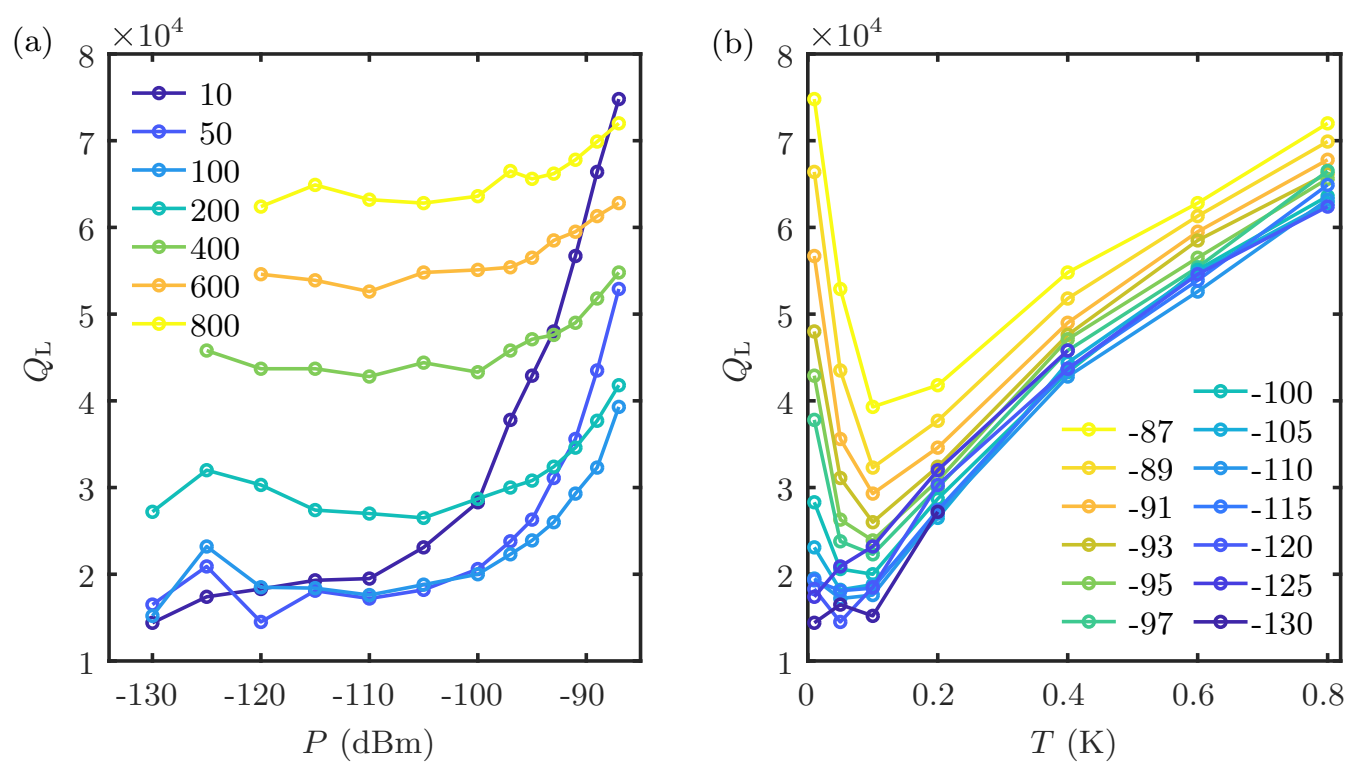

FIG. 10. Measured quality factor of sample B. (a) $Q_{\mathrm{L}}$ as a function of power at different bath temperatures as indicated in mK. The flux is $\Phi_{0} / 2$. (b) As (a) but the data are presented as a function of the bath temperature at different powers as indicated in $\mathrm{dBm}$. The lines are guides for the eye.

resonator is reduced to 200, which is of the order of the critical damping, photon numbers close to unity are obtained with an input power $P_{\mathrm{in}} \approx-85 \mathrm{dBm}$. Consequently, the photon number at the EP is well below unity in our experiments.

\section{APPENDIX F: SHIFT OF RESONANCE}

In Figs. 3, 6, and 7, the crossing of the mode frequencies as a function of the flux shifts slightly toward lower flux values with increasing bias voltage. We attribute this shift to heating of the SQUID, which results in a reduced critical current of the SQUID, and hence a larger inductance and lower resonance frequency of R2. The extracted critical currents are shown in Fig. 11. In principle, we vary also the Lamb shift [48], which, however, is not resolved since the resonance of R2 is very broad and, therefore, we neglect it in our model.
For simplicity, we do not take the frequency shift originating from the sample heating into account in the timedomain measurements in Fig. 5. The dependence of the damping rates on the frequency detuning decreases with increasing detuning, i.e., the surface in Fig. 2 is relatively flat with large $\delta$. Therefore, the effect of the heating is expected to be weak especially at the lowest pulse amplitudes. We note, however, that we observe a slight increase in the damping rate before the pulse with increasing pulse amplitudes. It may originate from an insufficient thermalization time between repeated measurements, resulting in an increased quasiparticle concentration in the sample. Nevertheless, the damping rate before the pulse increases from $2 \pi \times$ $0.16 \mathrm{MHz}$ only up to $2 \pi \times 0.21 \mathrm{MHz}$, which is substantially below the maximum damping rate of $2 \pi \times 0.39 \mathrm{MHz}$ in Fig. 5. Hence, the heating of the sample is of minor
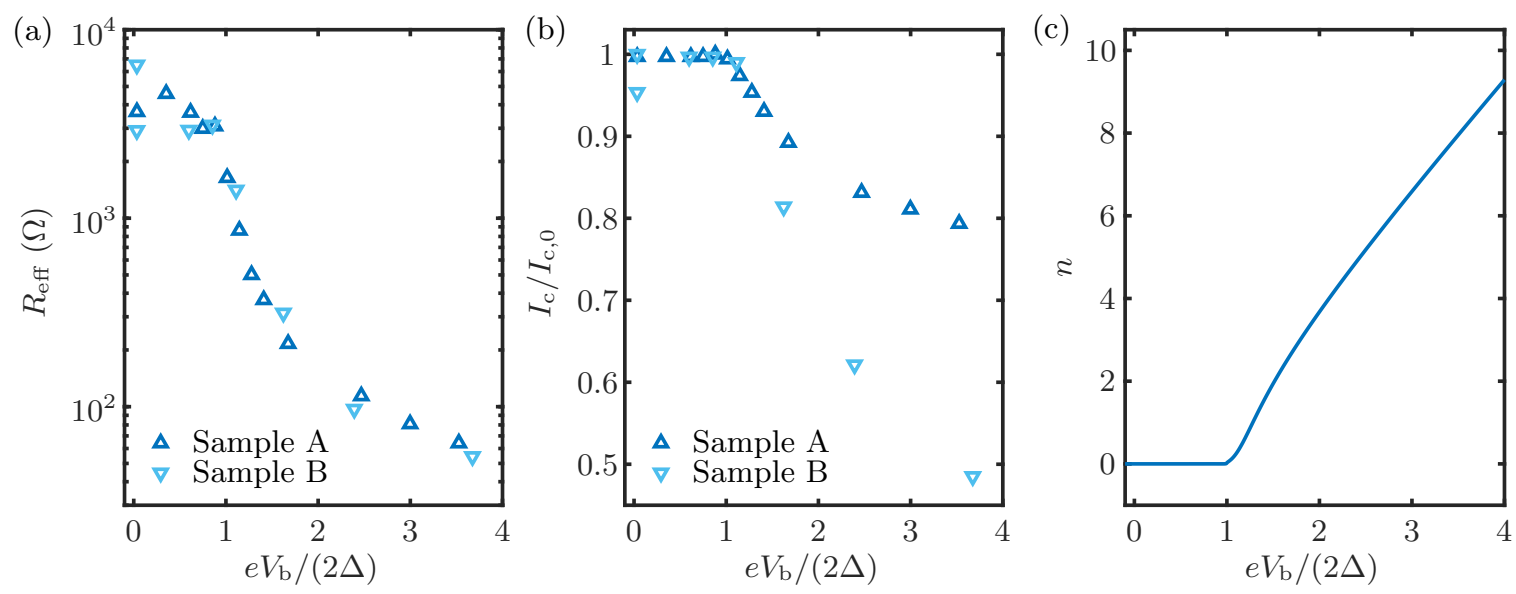

FIG. 11. Extracted parameters for the transition rates for samples A and B. (a) The effective resistance in the circuit model as a function of the bias voltage. (b) Critical current $I_{\mathrm{c}}$ normalized with the maximum critical current $I_{\mathrm{c}, 0}$. (c) Estimated average photon number in the dissipative resonator $\mathrm{R} 2$. 
TABLE I. Sample parameters. The parameters for sample B that differ from those for sample A are given in parenthesis. See Appendices E-I and Fig. 14(b) for details. The resonance frequency of the second mode of the resonator $\mathrm{R} 1, f_{1}$, is a measured value, the characteristic impedances of the transmission lines in the resonator, $Z_{0}$, and in the external measurement circuit, $Z_{\mathrm{L}}$, are nominal values. The lengths of the resonator sections $x_{1}$ and $x_{2}$ are design values, and the effective permittivity is calculated as [77] $\sqrt{\varepsilon_{\text {eff }}}=c /\left(2 f_{1} x_{1}\right)$, where $c$ is the speed of light in vacuum. We obtain the values for the capacitance per unit length $C_{\mathrm{l}}$, and the capacitance $C_{\mathrm{TL}}$ from finiteelement method (FEM) simulations. The capacitance $C_{\mathrm{C}}$ is obtained by fitting the circuit model to the measured scattering parameter $\left|S_{21}\right|$ in good agreement with FEM simulations, and the capacitances $C_{\mathrm{N}}, C_{\mathrm{S}}$, and $C_{\mathrm{j}}$ are calculated using a parallel-plate model. The coupling strength $g$ is obtained from $C_{\mathrm{C}}$. The loaded quality factor of the second mode of R1 $Q_{\mathrm{int}, 1}$ and the tunneling resistance $R_{\mathrm{T}}$ are measured values, and the Dynes parameter $\gamma_{\mathrm{D}}$ is estimated as the ratio of the asymptotic resistance and the zero-voltage resistance. The critical current at zero bias $I_{\mathrm{c}, 0}$ is given by the flux corresponding to the crossing of the modes in the circuit model in good agreement with a control sample with slightly smaller junction area and a critical current of approximately $200 \mathrm{nA}$. The damping rate $\kappa_{1}$ is given by the ratio $\omega_{1} / Q_{\text {int, } 1}$, and the damping rate $\kappa_{\text {int, } 2}$ is extracted from the saturation value of $\kappa_{2}$ at zero bias. The proportionality coefficient for the residual losses $\omega_{\mathrm{r}, \text { tot }}$ is a fitted value.

\begin{tabular}{lc}
\hline \hline Parameter & Value \\
\hline$f_{1}$ & $5.223 \mathrm{GHz}$ \\
$Z_{0}$ & $50 \Omega$ \\
$Z_{\mathrm{L}}$ & $50 \Omega$ \\
$x_{1}$ & $12 \mathrm{~mm}$ \\
$x_{2}$ & $6.0(6.5) \mathrm{mm}$ \\
$\varepsilon_{\text {eff }}$ & 5.73 \\
$C_{\mathrm{l}}$ & $155 \mathrm{pF} / \mathrm{m}$ \\
$C_{\mathrm{TL}}$ & $0.8 \mathrm{fF}$ \\
$C_{\mathrm{C}}$ & $3.8 \mathrm{fF}$ \\
$C_{\mathrm{N}}$ & $98 \mathrm{fF}$ \\
$C_{\mathrm{S}}$ & $460 \mathrm{fF}$ \\
$C_{\mathrm{j}}$ & $6.2 \mathrm{fF}$ \\
$g /(2 \pi)$ & $7.2 \mathrm{MHz}$ \\
$Q_{\text {int }, 1}$ & $10^{4}\left(2.0 \times 10^{4}\right)$ \\
$R_{\mathrm{T}}$ & $8.4(9.5) \mathrm{k} \Omega$ \\
$\gamma_{\mathrm{D}}$ & $1 \times 10^{-4}$ \\
$I_{\mathrm{c}, 0}$ & $340(300) \mathrm{nA}$ \\
$\kappa_{1} /(2 \pi)$ & $190(260) \mathrm{kHz}$ \\
$\kappa_{\text {int }, 2} /(2 \pi)$ & $16 \mathrm{MHz}$ \\
$\omega_{\mathrm{r}, \text { tot }} /(2 \pi)$ & $22 \mathrm{MHz}$ \\
\hline \hline
\end{tabular}

importance in the measurements of the damping rates during the QCR pulse.

\section{APPENDIX G: RESIDUAL LOSSES IN THE RESONATOR R2}

We attribute the residual voltage-dependent losses to dephasing, and to dissipation sources such as quasiparticle generation in the superconductors and resistive losses in the normal metal. First, the resonator R2 is slightly nonlinear owing to the SQUID and, hence, an increasing incoherent photon number results in dephasing. Dephasing can be added in Eq. (D2) with a term $\kappa_{\phi} \mathcal{L}\left[\hat{b}^{\dagger} \hat{b}\right] \hat{\rho}$, where the dephasing rate $\kappa_{\phi}$ depends on the number of thermal photons in the resonator. Similarly, in the case of superconducting qubits, the dephasing can be written as $\kappa_{\phi} \mathcal{L}\left[\hat{\sigma}_{z}\right] \hat{\rho}$, where $\hat{\sigma}_{z}$ is a Pauli operator. The factor $\kappa_{\phi}$ causes a similar effect as $\kappa_{2}$ in Eqs. (D2)-(D12) although it does not decrease the total photon number in the resonators. The photon-number variance for a thermal state is of the form [38,39] $n(n+1)$ and, therefore, thermal photons cause more dephasing than the coherent photons with a variance of $n$, where $n$ is the average photon number. Consequently, we assume that $\kappa_{\phi}=\omega_{\phi} n(n+1)$, where $\omega_{\phi}$ is a proportionality coefficient. Furthermore, as discussed above, the number of the coherent photons is low in R2 due to the relatively high loss rate. The steady-state photon number in $\mathrm{R} 2$ can be estimated as [49]

$$
n=\frac{\kappa_{\mathrm{QCR}} N_{\mathrm{QCR}}}{\kappa_{\mathrm{QCR}}+\kappa_{\mathrm{int}, 2}},
$$

where we assume that the photon number of the effective bath, to which R2 is coupled through $\kappa_{\text {int,2, }}$, vanishes owing to the very low cryostat temperatures of approximately $10 \mathrm{mK}$. The photon number $n$ depends linearly on the bias voltage at voltages above the superconductor energy gap, as shown in Fig. 11.

Second, we take the quasiparticle losses into account. The critical temperature of $\mathrm{Nb}$ is approximately $9 \mathrm{~K}$ and, therefore, the quasiparticle density remains low in it. However, the critical temperature of $\mathrm{Al}$ approximately $1.2 \mathrm{~K}$, which enables higher quasiparticle density than in $\mathrm{Nb}$. We observe a decrease in the critical current of the SQUID, which indicates increased temperature in the Al leads of the SQUID, and hence heat dissipation. The quasiparticle loss rate $[80,81]$ $\kappa_{\mathrm{qp}} \propto n_{\mathrm{qp}} \propto \sqrt{P}$, where $P$ is the absorbed power. The $\mathrm{Al}$ leads at the NIS junctions receive half of the Joule power $P=I V_{\mathrm{b}}$ at high voltages, whereas the other half is absorbed to the normal metal. Thus, the power is quadratic in voltage, which is linear in the estimated photon number. Therefore, the expected quasiparticle losses are linear in photon number $\kappa_{\mathrm{qp}}=\omega_{\mathrm{qp}} n$, where $\omega_{\mathrm{qp}}$ is a proportionality coefficient. The $\mathrm{dc}$ power dissipated in the junctions is substantially higher than the microwave input power. At $e V_{\mathrm{b}} /(2 \Delta)=2$, the dc power is approximately $30 \mathrm{pW}$ compared to a microwave power of $-100 \mathrm{dBm}=0.1 \mathrm{pW}$. The normal metal in the QCR acts as an effective quasiparticle trap [81] minimizing the quasiparticle losses. However, some fraction of the power dissipated at the QCR leaks to the SQUID.

There is an approximately $10-\mu \mathrm{m}$-long section of normal metal between the actual $\mathrm{Nb}$ resonator and the NIS junctions, which may cause some losses. The loss rate $\kappa_{\text {res }}$ at the resistor depends on the current profile of the microwave mode, which can depend on the voltage $V_{\mathrm{b}}$. Nevertheless, we assume these losses to be small since there is a layer of superconducting $\mathrm{Al}$ below the normal metal due to the shadow evaporation technique. This $\mathrm{Al}$ layer decreases the current in the resistor, and hence also the resistive losses. The very weak resistive losses are quadratic in the voltage amplitude of the microwave resonator which is linear in photon number if the mode profile does not change. Thus, the loss rate per photon $\kappa_{\text {res }}$ is approximately constant. 
Consequently, the total voltage-dependent losses in R2 including the dephasing, quasiparticle losses in the superconductors and the resistive losses are given by $\kappa_{\mathrm{r}, 2}=\kappa_{\phi}+$ $\kappa_{\mathrm{qp}}+\kappa_{\text {res }}$. We expect the dephasing to dominate over the quasiparticle and resistive losses. Therefore, in the numerical analysis, we take the photon-number-dependent losses into account as

$$
\kappa_{\mathrm{r}, 2}=\omega_{\mathrm{r}, \mathrm{tot}} n(n+1),
$$

with only one fitting parameter $\omega_{\mathrm{r}, \text { tot }}$ effectively describing the different loss methods discussed above. From the experimental damping rates of the dissipative resonator $\mathrm{R} 2$, we extract the the coefficient $\omega_{\mathrm{r} \text {,tot }} \approx 2 \pi \times 22 \mathrm{MHz}$. The good agreement with the experimental damping rate $\kappa_{2}$ and the model with the quadratic residual losses $\kappa_{\mathrm{r}, 2}$ in Fig. 4(a) gives further support for the approximation in Eq. (G2). We do not take this loss rate into account in Eq. (G1) for simplicity, and also due to the fact that pure dephasing does not decrease the photon number.

The odd modes of R1 do not show flux dependence as expected due to the voltage node at the coupling capacitor. However, they do show some dependence on the voltage $V_{\mathrm{b}}$. Similar dependence can be observed also for the even modes at $\Phi / \Phi_{0}=0.5$ where the inductance of the SQUID ideally vanishes and thus decouples the QCR from the resonator R1. We attribute this observation to unintentional asymmetry of the sample. Furthermore, the QCR may be weakly coupled to the input and output microwave fields through some spurious mode of the sample holder. The very broad resonance at high-bias voltages enables the coupling to the spurious modes. We note that the spurious modes may be partially responsible for the $\kappa_{\mathrm{r}, 2}$. However, we do not quantitatively model these losses. Instead, they are effectively included in the parameter $\omega_{\mathrm{r}, \text { tot }}$ in Eq. (G2).

\section{APPENDIX H: FULL MODEL FOR $\kappa_{2}$ AND $\kappa_{\mathrm{eff}}$}

The decay rate of the resonator $\mathrm{R} 2, \kappa_{2}$, and the effective damping rate of the coupled system, $\kappa_{\text {eff }}$, are obtained as follows. First, we extract the effective resistance corresponding to the QCR by fitting the classical circuit model to the experimentally obtained scattering parameter $S_{21}$ using a least-squares algorithm. In addition to the effective resistances, we extract the critical current of the SQUID from the theoretical model (Fig. 11). From the same fit, we also extract the coupling capacitance $C_{\mathrm{C}}$ at zero-bias voltage and assume it to be voltage independent throughout this work. The coupling capacitance is found to be $3.8 \mathrm{fF}$ which agrees well with the finite-element-method simulation that yields approximately $5 \mathrm{fF}$. The internal losses of R1 are extracted separately with R2 far detuned. Second, we calculate the quality factor of the resonator R2, $Q_{\mathrm{R} 2}$, for the obtained effective resistance, as discussed below. The coupling rate is related to the quality factor as $\kappa_{2}=\omega_{2} / Q_{\mathrm{R} 2}$. The full model shown in Fig. 4(a) is obtained by fitting

$$
\kappa_{2}\left(V_{\mathrm{b}}\right)=\kappa_{\mathrm{QCR}}\left(V_{\mathrm{b}}\right)+\kappa_{\mathrm{r}, 2}\left(V_{\mathrm{b}}\right)+\kappa_{\mathrm{int}, 2}
$$

to the experimental transition rates according to Eqs. (B8), (G1), and (G2). Here, we use $\omega_{\mathrm{r}, \text { tot }}$ as the only fitting parameter since we fix $\kappa_{\text {int,2 }}$ to the saturation value at zero bias, as discussed above, and $\kappa_{\mathrm{QCR}}$ is obtained from Eq. (B8) with parameters extracted from independent measurements.

Subsequently, we may proceed to the effective damping rates $\kappa_{\text {eff }}=-2 \operatorname{Im}\left(\lambda_{ \pm}\right)$, which can be obtained from $\kappa_{2}$ with the help of Eq. (2). The damping rate of R2 above the critical damping $\kappa_{2}>4 g$ results in the two branches of the effective damping rate $\kappa_{\text {eff }}$ at bias voltages $V_{\mathrm{b}} \gtrsim 2 \Delta / e$. We use Eq. (2) for both the experimental effective damping rates and the model in Fig. 4(b).

\section{APPENDIX I: CLASSICAL CIRCUIT MODEL}

To simulate the scattering parameter $S_{21}$, we use a classical circuit model similar to the one presented in Ref. [57]. We analyze the samples using standard microwave circuit theory [82]. The input impedance of the resonator $\mathrm{R} 2$ is

$$
Z_{\mathrm{R} 2}=Z_{\mathrm{C}}+\frac{Z_{0}\left\{Z_{\mathrm{S}}+Z_{0} \tanh \left(\gamma x_{2}\right)+\frac{Z_{0}\left[R_{\text {eff }}+Z_{0} \tanh \left(\gamma x_{2}\right)\right]}{Z_{0}+R_{\text {eff }} \tanh \left(\gamma x_{2}\right)}\right\}}{Z_{0}+\tanh \left(\gamma x_{2}\right)\left\{Z_{\mathrm{S}}+\frac{Z_{0}\left[R_{\text {eff }}+Z_{0} \tanh \left(\gamma x_{2}\right)\right]}{Z_{0}+R_{\text {eff }} \tanh \left(\gamma x_{2}\right)}\right\}},
$$

where the impedance of the SQUID and the capacitors between the SQUID and the center conductor is given by $Z_{\mathrm{S}}=$ $i \omega L_{\mathrm{S}}+2 /\left(i \omega C_{\mathrm{S}}\right)$, the impedance of the coupling capacitor between the resonators by $Z_{\mathrm{C}}=1 /\left(i \omega C_{\mathrm{C}}\right), \gamma$ is the complex propagation coefficient discussed above, and the terminating impedance consisting of the effective resistance of the NIS junctions and the capacitor between the normal-metal island and the center conductor is modeled as an effective resistor with resistance $R_{\text {eff. }}$. The inductance of the SQUID is calculated as $L_{\mathrm{S}}(\Phi)=\Phi_{0} /\left[2 \pi I_{0}\left|\cos \left(\pi \Phi / \Phi_{0}\right)\right|\right]$, where the maximum supercurrent through the SQUID is $I_{0}$, and the flux quantum is $\Phi_{0}=h /(2 e)$.

The scattering parameter $S_{21}$ describing the voltage transmission from port 1 to port 2 can be calculated using the transmission matrix method as [82]

$$
S_{21}=\frac{2}{A_{\mathrm{m}}+B_{\mathrm{m}} / Z_{\mathrm{L}}+C_{\mathrm{m}} Z_{\mathrm{L}}+D_{\mathrm{m}}},
$$

where $Z_{\mathrm{L}}$ is the characteristic impedance of the external measurement cables, and

$$
\left(\begin{array}{ll}
A_{\mathrm{m}} & B_{\mathrm{m}} \\
C_{\mathrm{m}} & D_{\mathrm{m}}
\end{array}\right)=M_{1} M_{2} M_{3} M_{2} M_{1},
$$

with

$$
\begin{gathered}
M_{1}=\left(\begin{array}{cc}
1 & \frac{1}{i \omega C_{\mathrm{TL}}} \\
0 & 1
\end{array}\right), \\
M_{2}=\left(\begin{array}{cc}
\cosh \left(\gamma x_{1}\right) & Z_{0} \sinh \left(\gamma x_{1}\right) \\
\frac{1}{Z_{0}} \sinh \left(\gamma x_{1}\right) & \cosh \left(\gamma x_{1}\right)
\end{array}\right), \\
M_{3}=\left(\begin{array}{cc}
1 & 0 \\
\frac{1}{Z_{\mathrm{R} 2}} & 1
\end{array}\right) .
\end{gathered}
$$

We analyze the losses in the resonator R2 also in the absence of coupling to R1 as shown in Fig. 12. In this case, we omit matrices $M_{1}$ and $M_{2}$ from Eq. (I3). The resonator R2 causes a dip in the amplitude of the transmission coefficient $S_{21}$, whereas R1 causes a peak. The quality factor can be estimated directly from the ratio of the center frequency and the width of the peak or dip. Alternatively, more advanced methods can be 


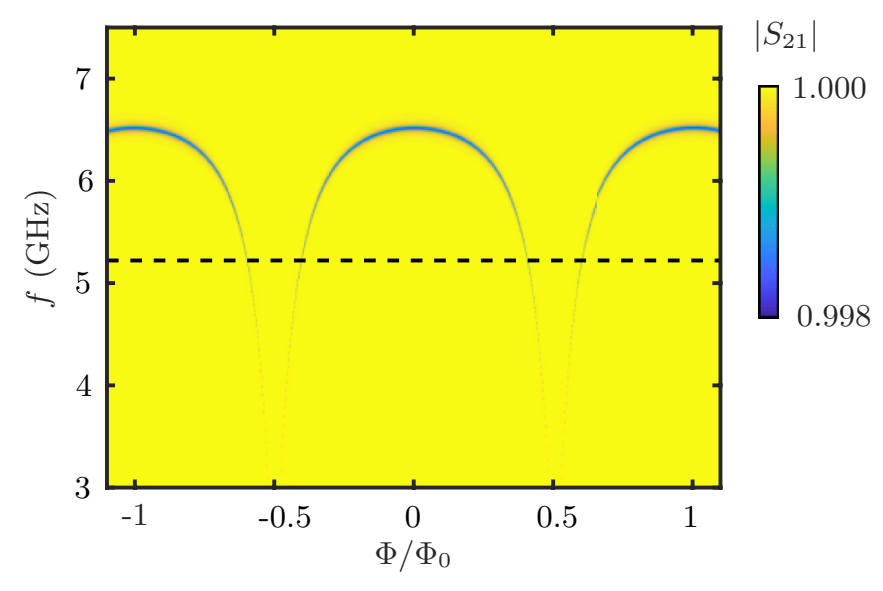

FIG. 12. Simulation of the scattering parameter of resonator R2. The scattering parameter is presented as a function of magnetic flux and frequency. We omit R1 in the simulation by letting $C_{\mathrm{TL}} \rightarrow \infty$. For clarity, we show the flux-independent resonance frequency of R1 as the dashed line. Near the mode crossing at $\Phi \approx 0.4 \times \Phi_{0}$, a flux detuning of $0.01 \times \Phi_{0}$ results in a frequency detuning of approximately $140 \mathrm{MHz}$. The simulation parameters correspond to sample B.

used [83]. In addition, these simulations yield the frequency detuning as a function the magnetic flux.

\section{APPENDIX J: SAMPLE FABRICATION}

The samples are fabricated on a Si wafer with a thickness of $500 \mu \mathrm{m}$ and a diameter of $100 \mathrm{~mm}$. First, a 300-nm-thick layer of $\mathrm{SiO}_{2}$ is thermally grown on the wafer with resistivity $\rho>10 \mathrm{k} \Omega \mathrm{cm}$. Subsequently, a $200-\mathrm{nm}$-thick layer of $\mathrm{Nb}$ is sputtered on top of the oxide. The resonators are patterned on the $\mathrm{Nb}$ layer with optical lithography and reactive ion etching. We cover the complete wafer with a 40-nm-thick layer of $\mathrm{Al}_{2} \mathrm{O}_{3}$ fabricated using atomic-layer deposition. This oxide layer serves as an insulating barrier in the parallel plate capacitors and separates the QCR lines from the ground plane. The nanostructures are defined using electron beam lithography and two-angle shadow evaporation followed by a lift-off process. The SQUID consists of two Al layers with thicknesses of $40 \mathrm{~nm}$ each. The first Al layer is oxidized in situ in the evaporation chamber at $1.0 \mathrm{mbar}$ for $5 \mathrm{~min}$. The
SINIS junctions consist of $\mathrm{Al}(40 \mathrm{~nm})$ and $\mathrm{Cu}(40 \mathrm{~nm})$, and the Al layer is similarly oxidized as in the SQUID. The shadow evaporation technique results in overlapping metal layers. Scanning electron micrographs of the samples are shown in Fig. 13.

\section{APPENDIX K: MEASUREMENT SETUP}

The measurement setup is schematically presented in Fig. 14. The samples are measured in a commercial dry dilution refrigerator with a base temperature of approximately $10 \mathrm{mK}$. To characterize the samples, we use standard microwave techniques that are well established in the field of circuit quantum electrodynamics. The scattering parameters are measured with a vector network analyzer which contains both the microwave source and the detector. The microwave signal is attenuated at different temperature stages to avoid heat leakage from higher temperatures to the sample. We employ amplifiers at $4 \mathrm{~K}$ and at room temperature. The NIS junctions are controlled by applying a bias voltage or current through continuous thermocoax cables from room temperature down to the base temperature. Magnetic flux for the SQUID is produced using a superconducting coil with a bias current.

The time-domain measurements are carried out using a field programmable gate array with a measurement setup resembling the one used in Ref. [23]. The voltage pulses to the QCR are produced with an arbitrary waveform generator. We obtain an accurate in situ calibration of the attenuation in the measurement cables leading to the QCR from the rapid increase of the damping rate with increasing pulse amplitudes at $e V_{\mathrm{b}} /(2 \Delta)=1$.

\section{APPENDIX L: NORMALIZATION OF SCATTERING PARAMETERS}

All measured scattering parameters $S_{21}$ are normalized. Initially, we compensate for the phase winding originating from the electrical delay $\tau \approx 50 \mathrm{~ns}$ in the measurement setup outside the sample by multiplying the measured transmission coefficient by $\exp (i \omega \tau)$. Consequently, the resonance produces a circle on the complex plane as the frequency is increased over the resonance. We transform this circle to its canonical position where $\max \left|S_{21}\right|$ is on the positive real axis and the circle intersects the origin. Finally, we normalize the amplitude to unity by dividing with $\max \left|S_{21}\right|$. (a)

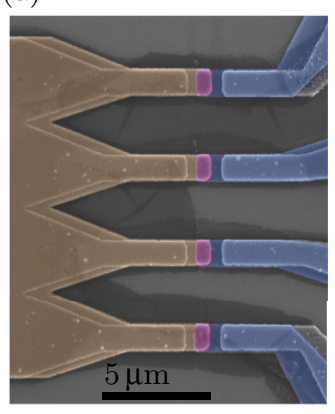

(b)

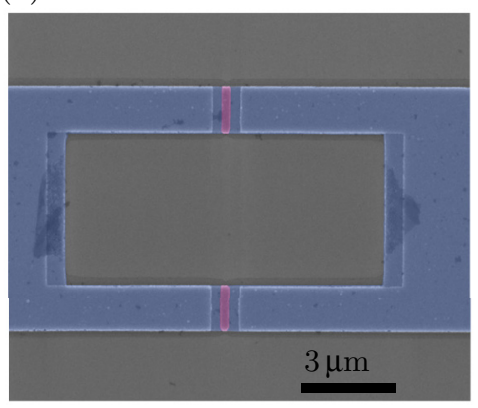

(c)

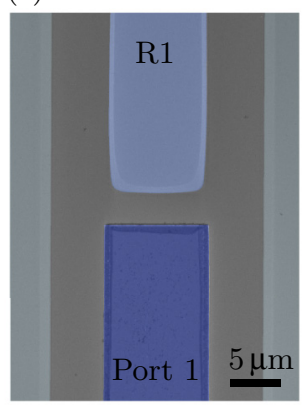

(d)

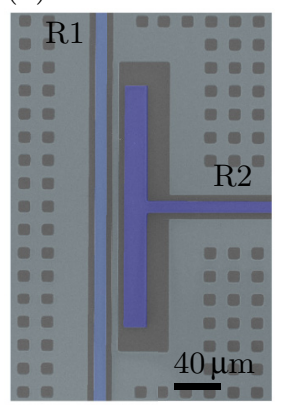

FIG. 13. False-color scanning electron micrographs showing sample structure. (a) Normal-metal island with four NIS junctions. (b) SQUID with two Josephson junctions. (c) Coupling capacitance between R1 and port 1. (d) Coupling capacitance between R1 and R2. 


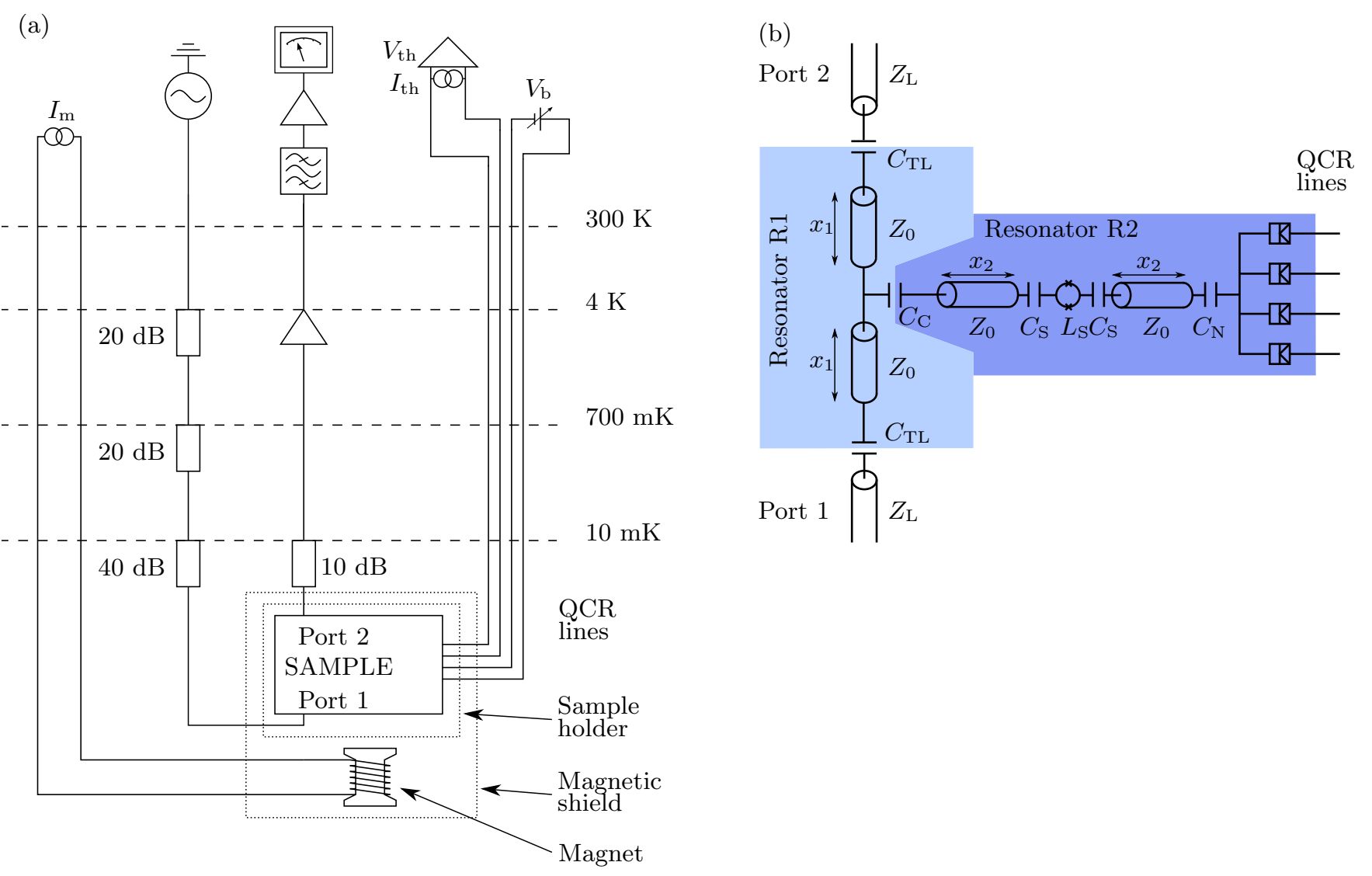

FIG. 14. Measurement setup and circuit diagram of the sample. (a) Simplified measurement setup showing the attenuators, and amplifiers at different temperatures. We measure the sample response to microwave signal from port 1 to port 2. Magnetic field for the SQUID is generated using a coil with current $I_{\mathrm{m}}$. A bias voltage $V_{\mathrm{b}}$ and bias current for thermometry $I_{\mathrm{th}}$ are applied to the NIS junctions. The temperature of the normal metal is deduced from voltage $V_{\text {th }}$ measured with an applied bias current $I_{\text {th }}$. (b) Sample structure presented as an electrical circuit diagram. The transmission lines of the resonators have characteristic impedances $Z_{0}$, and the external transmission lines $Z_{\mathrm{L}}$. The sections of the resonators have lengths $x_{1}$ and $x_{2}$. The capacitances at the external ports are denoted by $C_{\mathrm{TL}}$, between the resonators by $C_{\mathrm{C}}$, between the SQUID with inductance $L_{\mathrm{S}}$ and the center conductor of the transmission line by $C_{\mathrm{S}}$, and between the normal-metal island and the center conductor by $C_{\mathrm{N}}$.

[1] T. J. Milburn, J. Doppler, C. A. Holmes, S. Portolan, S. Rotter, and P. Rabl, General description of quasiadiabatic dynamical phenomena near exceptional points, Phys. Rev. A 92, 052124 (2015).

[2] H. Xu, D. Mason, L. Jiang, and J. G. E. Harris, Topological energy transfer in an optomechanical system with exceptional points, Nature (London) 537, 80 (2016).

[3] J. Doppler, A. A. Mailybaev, J. Böhm, U. Kuhl, A. Girschik, F. Libisch, T. J. Milburn, P. Rabl, N. Moiseyev, and S. Rotter, Dynamically encircling an exceptional point for asymmetric mode switching, Nature (London) 537, 76 (2016).

[4] I. Mandal and S. Tewari, Exceptional point description of onedimensional chiral topological superconductors/superfluids in BDI class, Physica E (Amsterdam) 79, 180 (2016).

[5] K. Ding, G. Ma, M. Xiao, Z. Q. Zhang, and C. T. Chan, Emergence, Coalescence, and Topological Properties of Multiple Exceptional Points and their Experimental Realization, Phys. Rev. X 6, 021007 (2016).
[6] K. Ding, G. Ma, Z. Q. Zhang, and C. T. Chan, Experimental Demonstration of an Anisotropic Exceptional Point, Phys. Rev. Lett. 121, 085702 (2018).

[7] P. San-Jose, J. Cayao, E. Prada, and R. Aguado, Majorana bound states from exceptional points in non-topological superconductors, Sci. Rep. 6, 21427 (2016).

[8] T. Kato, Perturbation Theory for Linear Operators, Grundlehren der mathematischen Wissenschaften, Vol. 132 (Springer, Berlin, 1966).

[9] C. Dembowski, H.-D. Gräf, H. L. Harney, A. Heine, W. D. Heiss, H. Rehfeld, and A. Richter, Experimental Observation of the Topological Structure of Exceptional Points, Phys. Rev. Lett. 86, 787 (2001).

[10] W. Heiss, Exceptional points-their universal occurrence and their physical significance, Czech. J. Phys. 54, 1091 (2004).

[11] M. Berry, Physics of non-Hermitian degeneracies, Czech. J. Phys. 54, 1039 (2004).

[12] W. D. Heiss, The physics of exceptional points, J. Phys. A: Math. Theor. 45, 444016 (2012). 
[13] H. Cao and J. Wiersig, Dielectric microcavities: Model systems for wave chaos and non-Hermitian physics, Rev. Mod. Phys. 87, 61 (2015).

[14] B. Dietz, T. Friedrich, J. Metz, M. Miski-Oglu, A. Richter, F. Schäfer, and C. A. Stafford, Rabi oscillations at exceptional points in microwave billiards, Phys. Rev. E 75, 027201 (2007).

[15] C. Dembowski, B. Dietz, H.-D. Gräf, H. L. Harney, A. Heine, W. D. Heiss, and A. Richter, Observation of a Chiral State in a Microwave Cavity, Phys. Rev. Lett. 90, 034101 (2003).

[16] C. M. Bender and S. Boettcher, Real Spectra in Non-Hermitian Hamiltonians Having $\mathcal{P} \mathcal{T}$ Symmetry, Phys. Rev. Lett. 80, 5243 (1998).

[17] A. Guo, G. J. Salamo, D. Duchesne, R. Morandotti, M. Volatier-Ravat, V. Aimez, G. A. Siviloglou, and D. N. Christodoulides, Observation of $\mathcal{P} \mathcal{T}$-Symmetry Breaking in Complex Optical Potentials, Phys. Rev. Lett. 103, 093902 (2009).

[18] N. M. Chtchelkatchev, A. A. Golubov, T. I. Baturina, and V. M. Vinokur, Stimulation of the Fluctuation Superconductivity by $\mathcal{P} \mathcal{T}$ Symmetry, Phys. Rev. Lett. 109, 150405 (2012).

[19] R. El-Ganainy, K. G. Makris, M. Khajavikhan, Z. H. Musslimani, S. Rotter, and D. N. Christodoulides, NonHermitian physics and PT symmetry, Nat. Phys. 14, 11 (2018).

[20] K. V. Kepesidis, T. J. Milburn, J. Huber, K. G. Makris, S. Rotter, and P. Rabl, $\mathcal{P} \mathcal{T}$-symmetry breaking in the steady state of microscopic gain-loss systems, New J. Phys. 18, 095003 (2016)

[21] F. Quijandría, U. Naether, S. K. Özdemir, F. Nori, and D. Zueco, $\mathcal{P} \mathcal{T}$-symmetric circuit qed, Phys. Rev. A 97, 053846 (2018).

[22] K. Inomata, Z. Lin, K. Koshino, W. D. Oliver, J.-S. Tsai, T. Yamamoto, and Y. Nakamura, Single microwave-photon detector using an artificial $\Lambda$-type three-level system, Nat. Commun. 7, 12303 (2016).

[23] J. Govenius, R. E. Lake, K. Y. Tan, and M. Möttönen, Detection of Zeptojoule Microwave Pulses Using Electrothermal Feedback in Proximity-Induced Josephson Junctions, Phys. Rev. Lett. 117, 030802 (2016).

[24] J. Ding, P. A. R. Ade, A. J. Anderson, J. Avva, Z. Ahmed, K. Arnold, J. E. Austermann, A. N. Bender, B. A. Benson, L. E. Bleem et al., Optimization of transition edge sensor arrays for cosmic microwave background observations with the South Pole Telescope, IEEE T. Appl. Supercon. 27, 1 (2017).

[25] A. Opremcak, I. V. Pechenezhskiy, C. Howington, B. G. Christensen, M. A. Beck, E. Leonard, J. Suttle, C. Wilen, K. N. Nesterov, G. J. Ribeill, T. Thorbeck, F. Schlenker, M. G. Vavilov, B. L. T. Plourde, and R. McDermott, Measurement of a superconducting qubit with a microwave photon counter, Science 361, 1239 (2018).

[26] T. D. Ladd, F. Jelezko, R. Laflamme, Y. Nakamura, C. Monroe, and J. L. O'Brien, Quantum computers, Nature (London) 464, 45 (2010).

[27] J. Clarke and F. K. Wilhelm, Superconducting quantum bits, Nature (London) 453, 1031 (2008).

[28] I. M. Georgescu, S. Ashhab, and F. Nori, Quantum simulation, Rev. Mod. Phys. 86, 153 (2014).

[29] A. Blais, R.-S. Huang, A. Wallraff, S. M. Girvin, and R. J. Schoelkopf, Cavity quantum electrodynamics for superconducting electrical circuits: An architecture for quantum computation, Phys. Rev. A 69, 062320 (2004).
[30] A. Wallraff, D. I. Schuster, A. Blais, L. Frunzio, R.-S. Huang, J. Majer, S. Kumar, S. M. Girvin, and R. J. Schoelkopf, Strong coupling of a single photon to a superconducting qubit using circuit quantum electrodynamics, Nature (London) 431, 162 (2004).

[31] R. Barends, J. Kelly, A. Megrant, A. Veitia, D. Sank, E. Jeffrey, T. C. White, J. Mutus, A. G. Fowler, B. Campbell, Y. Chen, Z. Chen, B. Chiaro, A. Dunsworth, C. Neill, P. O’Malley, P. Roushan, A. Vainsencher, J. Wenner, A. N. Korotkov et al., Superconducting quantum circuits at the surface code threshold for fault tolerance, Nature (London) 508, 500 (2014).

[32] J. Kelly, R. Barends, A. G. Fowler, A. Megrant, E. Jeffrey, T. C. White, D. Sank, J. Y. Mutus, B. Campbell, Y. Chen, Z. Chen, B. Chiaro, A. Dunsworth, I.-C. Hoi, C. Neill, P. J. J. O’Malley, C. Quintana, P. Roushan, A. Vainsencher, J. Wenner et al., State preservation by repetitive error detection in a superconducting quantum circuit, Nature (London) 519, 66 (2015).

[33] Edited by D. Lidar and T. Brun, Quantum Error Correction (Cambridge University Press, Cambridge, 2013).

[34] B. M. Terhal, Quantum error correction for quantum memories, Rev. Mod. Phys. 87, 307 (2015).

[35] J. Q. You and F. Nori, Atomic physics and quantum optics using superconducting circuits, Nature (London) 474, 589 (2011).

[36] M. H. Devoret and R. J. Schoelkopf, Superconducting circuits for quantum information: An outlook, Science 339, 1169 (2013).

[37] G. Wendin, Quantum information processing with superconducting circuits: A review, Rep. Prog. Phys. 80, 106001 (2017).

[38] A. A. Clerk and D. W. Utami, Using a qubit to measure photonnumber statistics of a driven thermal oscillator, Phys. Rev. A 75, 042302 (2007).

[39] J. Goetz, S. Pogorzalek, F. Deppe, K. G. Fedorov, P. Eder, M. Fischer, F. Wulschner, E. Xie, A. Marx, and R. Gross, Photon Statistics of Propagating Thermal Microwaves, Phys. Rev. Lett. 118, 103602 (2017).

[40] K. Geerlings, Z. Leghtas, I. M. Pop, S. Shankar, L. Frunzio, R. J. Schoelkopf, M. Mirrahimi, and M. H. Devoret, Demonstrating a Driven Reset Protocol for a Superconducting Qubit, Phys. Rev. Lett. 110, 120501 (2013).

[41] C. C. Bultink, M. A. Rol, T. E. O’Brien, X. Fu, B. C. S. Dikken, C. Dickel, R. F. L. Vermeulen, J. C. de Sterke, A. Bruno, R. N. Schouten, and L. DiCarlo, Active Resonator Reset in the Nonlinear Dispersive Regime of Circuit QED, Phys. Rev. Appl. 6, 034008 (2016).

[42] C. H. Wong, C. Wilen, R. McDermott, and M. G. Vavilov, A tunable quantum dissipator for active resonator reset in circuit QED, Quantum Sci. Technol. 4, 025001 (2019).

[43] D. T. McClure, H. Paik, L. S. Bishop, M. Steffen, J. M. Chow, and J. M. Gambetta, Rapid Driven Reset of a Qubit Readout Resonator, Phys. Rev. Appl. 5, 011001 (2016).

[44] S. Boutin, C. K. Andersen, J. Venkatraman, A. J. Ferris, and A. Blais, Resonator reset in circuit qed by optimal control for large open quantum systems, Phys. Rev. A 96, 042315 (2017).

[45] J. Tuorila, M. Partanen, T. Ala-Nissila, and M. Möttönen, Efficient protocol for qubit initialization with a tunable environment, npj Quantum Inf. 3, 27 (2017).

[46] K. Y. Tan, M. Partanen, R. E. Lake, J. Govenius, S. Masuda, and M. Möttönen, Quantum-circuit refrigerator, Nat. Commun. 8, 15189 (2017). 
[47] S. Masuda, K. Y. Tan, M. Partanen, R. E. Lake, J. Govenius, M. Silveri, H. Grabert, and M. Möttönen, Observation of microwave absorption and emission from incoherent electron tunneling through a normal-metal-insulator-superconductor junction, Sci. Rep. 8, 3966 (2018).

[48] M. Silveri, S. Masuda, V. Sevriuk, K. Y. Tan, M. Jenei, E. Hyyppä, F. Hassler, M. Partanen, J. Goetz, R. E. Lake, L. Grönberg, and M. Möttönen, Broadband Lamb shift in an engineered quantum system, Nat. Phys. 15, 533 (2019).

[49] M. Silveri, H. Grabert, S. Masuda, K. Y. Tan, and M. Möttönen, Theory of quantum-circuit refrigeration by photon-assisted electron tunneling, Phys. Rev. B 96, 094524 (2017).

[50] Z. L. Wang, Y. P. Zhong, L. J. He, H. Wang, J. M. Martinis, A. N. Cleland, and Q. W. Xie, Quantum state characterization of a fast tunable superconducting resonator, Appl. Phys. Lett. 102, 163503 (2013).

[51] M. Pierre, I.-M. Svensson, S. R. Sathyamoorthy, G. Johansson, and P. Delsing, Storage and on-demand release of microwaves using superconducting resonators with tunable coupling, Appl. Phys. Lett. 104, 232604 (2014).

[52] A. Baust, E. Hoffmann, M. Haeberlein, M. J. Schwarz, P. Eder, J. Goetz, F. Wulschner, E. Xie, L. Zhong, F. Quijandría, B. Peropadre, D. Zueco, J.-J. García Ripoll, E. Solano, K. Fedorov, E. P. Menzel, F. Deppe, A. Marx, and R. Gross, Tunable and switchable coupling between two superconducting resonators, Phys. Rev. B 91, 014515 (2015).

[53] M. R. Vissers, J. Hubmayr, M. Sandberg, S. Chaudhuri, C. Bockstiegel, and J. Gao, Frequency-tunable superconducting resonators via nonlinear kinetic inductance, Appl. Phys. Lett. 107, 062601 (2015).

[54] F. Wulschner, J. Goetz, F. R. Koessel, E. Hoffmann, A. Baust, P. Eder, M. Fischer, M. Haeberlein, M. J. Schwarz, M. Pernpeintner, E. Xie, L. Zhong, C. W. Zollitsch, B. Peropadre, J.-J. Garcia Ripoll, E. Solano, K. G. Fedorov, E. P. Menzel, F. Deppe, A. Marx, and R. Gross, Tunable coupling of transmission-line microwave resonators mediated by an $\mathrm{rf}$ SQUID, EPJ Quantum Technol. 3, 10 (2016).

[55] A. A. Adamyan, S. E. Kubatkin, and A. V. Danilov, Tunable superconducting microstrip resonators, Appl. Phys. Lett. 108, 172601 (2016).

[56] M. Pierre, S. R. Sathyamoorthy, I.-M. Svensson, G. Johansson, and P. Delsing, Resonant and off-resonant microwave signal manipulation in coupled superconducting resonators, Phys. Rev. B 99, 094518 (2019).

[57] M. Partanen, K. Y. Tan, S. Masuda, J. Govenius, R. E. Lake, M. Jenei, L. Grönberg, J. Hassel, S. Simbierowicz, V. Vesterinen, J. Tuorila, T. Ala-Nissila, and M. Möttönen, Flux-tunable heat sink for quantum electric circuits, Sci. Rep. 8, 6325 (2018).

[58] N. G. Dickson, M. W. Johnson, M. H. Amin, R. Harris, F. Altomare, A. J. Berkley, P. Bunyk, J. Cai, E. M. Chapple, P. Chavez, F. Cioata, T. Cirip, P. deBuen, M. Drew-Brook, C. Enderud, S. Gildert, F. Hamze, J. P. Hilton, E. Hoskinson, K. Karimi et al., Thermally assisted quantum annealing of a 16-qubit problem, Nat. Commun. 4, 1903 (2013).

[59] R. Nandkishore and D. A. Huse, Many-body localization and thermalization in quantum statistical mechanics, Annu. Rev. Condens. Matter Phys. 6, 15 (2015).

[60] A. Metelmann and H. E. Türeci, Nonreciprocal signal routing in an active quantum network, Phys. Rev. A 97, 043833 (2018).
[61] A. Mäkinen, J. Ikonen, M. Partanen, and M. Möttönen, Reconstruction approach to quantum dynamics of bosonic systems, arXiv:1904.11353 [Phys. Rev. A (to be published)].

[62] M. D. Reed, B. R. Johnson, A. A. Houck, L. DiCarlo, J. M. Chow, D. I. Schuster, L. Frunzio, and R. J. Schoelkopf, Fast reset and suppressing spontaneous emission of a superconducting qubit, Appl. Phys. Lett. 96, 203110 (2010).

[63] V. A. Sevriuk, K. Y. Tan, E. Hyyppä, M. Silveri, M. Partanen, M. Jenei, S. Masuda, J. Goetz, V. Vesterinen, L. Grönberg, and M. Möttönen, Fast control of dissipation in a superconducting resonator, Appl. Phys. Lett. 115, 082601 (2019).

[64] G. Demange and E.-M. Graefe, Signatures of three coalescing eigenfunctions, J. Phys. A: Math. Theor. 45, 025303 (2012).

[65] F. Yoshihara, T. Fuse, S. Ashhab, K. Kakuyanagi, S. Saito, and K. Semba, Superconducting qubit-oscillator circuit beyond the ultrastrong-coupling regime, Nat. Phys. 13, 44 (2016).

[66] S. Barzanjeh, M. Wulf, M. Peruzzo, M. Kalaee, P. B. Dieterle, O. Painter, and J. M. Fink, Mechanical on-chip microwave circulator, Nat. Commun. 8, 953 (2017).

[67] S. Barzanjeh, M. Aquilina, and A. Xuereb, Manipulating the Flow of Thermal Noise in Quantum Devices, Phys. Rev. Lett. 120, 060601 (2018).

[68] M. Naghiloo, M. Abbasi, Y. N. Joglekar, and K. W. Murch, Quantum state tomography across the exceptional point in a single dissipative qubit, arXiv:1901.07968.

[69] M. Nahum, T. M. Eiles, and J. M. Martinis, Electronic microrefrigerator based on a normal-insulator-superconductor tunnel junction, Appl. Phys. Lett. 65, 3123 (1994).

[70] M. M. Leivo, J. P. Pekola, and D. V. Averin, Efficient Peltier refrigeration by a pair of normal metal/insulator/superconductor junctions, Appl. Phys. Lett. 68, 1996 (1996).

[71] F. Giazotto, T. T. Heikkilä, A. Luukanen, A. M. Savin, and J. P. Pekola, Opportunities for mesoscopics in thermometry and refrigeration: Physics and applications, Rev. Mod. Phys. 78, 217 (2006).

[72] A. V. Feshchenko, L. Casparis, I. M. Khaymovich, D. Maradan, O.-P. Saira, M. Palma, M. Meschke, J. P. Pekola, and D. M. Zumbühl, Tunnel-Junction Thermometry Down to Millikelvin Temperatures, Phys. Rev. Appl. 4, 034001 (2015).

[73] A. Ronzani, B. Karimi, J. Senior, Y.-C. Chang, J. T. Peltonen, C. Chen, and J. P. Pekola, Tunable photonic heat transport in a quantum heat valve, Nat. Phys. 14, 991 (2018).

[74] A. Fornieri and F. Giazotto, Towards phase-coherent caloritronics in superconducting circuits, Nat. Nanotechnol. 12, 944 (2017).

[75] J. P. Pekola, V. F. Maisi, S. Kafanov, N. Chekurov, A. Kemppinen, Y. A. Pashkin, O.-P. Saira, M. Möttönen, and J. S. Tsai, Environment-Assisted Tunneling as an Origin of the Dynes Density of States, Phys. Rev. Lett. 105, 026803 (2010).

[76] P. J. Jones, J. Salmilehto, and M. Möttönen, Highly controllable qubit-bath coupling based on a sequence of resonators, J. Low Temp. Phys. 173, 152 (2013).

[77] M. Göppl, A. Fragner, M. Baur, R. Bianchetti, S. Filipp, J. M. Fink, P. J. Leek, G. Puebla, L. Steffen, and A. Wallraff, Coplanar waveguide resonators for circuit quantum electrodynamics, J. Appl. Phys. 104, 113904 (2008).

[78] J. Zmuidzinas, Superconducting microresonators: Physics and applications, Annu. Rev. Condens. Matter Phys. 3, 169 (2012). 
[79] G. Oelsner, C. K. Andersen, M. Rehák, M. Schmelz, S. Anders, M. Grajcar, U. Hübner, K. Mølmer, and E. Il'ichev, Detection of Weak Microwave Fields with an Underdamped Josephson Junction, Phys. Rev. Appl. 7, 014012 (2017).

[80] R. Barends, J. Wenner, M. Lenander, Y. Chen, R. C. Bialczak, J. Kelly, E. Lucero, P. O’Malley, M. Mariantoni, D. Sank, H. Wang, T. C. White, Y. Yin, J. Zhao, A. N. Cleland, J. M. Martinis, and J. J. A. Baselmans, Minimizing quasiparticle generation from stray infrared light in superconducting quantum circuits, Appl. Phys. Lett. 99, 113507 (2011).
[81] G. C. O'Neil, P. J. Lowell, J. M. Underwood, and J. N. Ullom, Measurement and modeling of a large-area normal-metal/insulator/superconductor refrigerator with improved cooling, Phys. Rev. B 85, 134504 (2012).

[82] D. Pozar, Microwave Engineering, 4th ed. (Wiley, Hoboken, NJ, 2011).

[83] P. J. Petersan and S. M. Anlage, Measurement of resonant frequency and quality factor of microwave resonators: Comparison of methods, J. Appl. Phys. 84, 3392 (1998). 